\title{
COMPUTATIONS OF EISENSTEIN SERIES ON FUCHSIAN GROUPS
}

\author{
HELEN AVELIN
}

\begin{abstract}
We present numerical investigations of the value distribution and distribution of Fourier coefficients of the Eisenstein series $E(z ; s)$ on arithmetic and non-arithmetic Fuchsian groups. Our numerics indicate a Gaussian limit value distribution for a real-valued rotation of $E(z ; s)$ as $\operatorname{Re} s=1 / 2, \operatorname{Im} s \rightarrow \infty$ and also, on non-arithmetic groups, a complex Gaussian limit distribution for $E(z ; s)$ when $\operatorname{Re} s>1 / 2$ near $1 / 2$ and $\operatorname{Im} s \rightarrow \infty$, at least if we allow $\operatorname{Re} s \rightarrow 1 / 2$ at some rate. Furthermore, on non-arithmetic groups and for fixed $s$ with $\operatorname{Re} s \geq 1 / 2$ near $1 / 2$, our numerics indicate a Gaussian limit distribution for the appropriately normalized Fourier coefficients.
\end{abstract}

\section{INTRODUCTION}

The study of eigenfunctions of the Laplacian on a negatively curved Riemannian manifold is of high significance in the research field of quantum chaos. The $L^{2}$ normalized eigenfunctions $\phi_{n}(z)$ represent individual quantum mechanical particles, and eigenfunctions with large eigenvalue $\lambda_{n}$ are of particular interest. It is in the semi-classical limit $\lambda_{n} \rightarrow \infty$ (which corresponds to $\hbar \rightarrow 0$ in Schrödinger's equation) that one has the opportunity to study the impact of chaos in classical dynamics on quantum mechanical systems.

The surfaces considered in this paper are hyperbolic surfaces; we will always write them as $\Gamma \backslash \mathcal{H}$, where $\Gamma$ is a cofinite Fuchsian group acting on the Poincaré upper half-plane $\mathcal{H}$ equipped with the hyperbolic metric $d s^{2}=y^{-2}\left(d x^{2}+d y^{2}\right)$ and corresponding area $d \mu=d x d y / y^{2}$. When the surface $\Gamma \backslash \mathcal{H}$ is of finite area but non-compact, i.e., has cusps, Selberg Sel89] showed that the Laplace-Beltrami operator

$$
\Delta=y^{2}\left(\frac{\partial^{2}}{\partial x^{2}}+\frac{\partial^{2}}{\partial y^{2}}\right)
$$

has both a discrete and a continuous spectrum and that the continuous spectrum is the interval $[1 / 4, \infty)$. The discrete eigenfunctions with $1 / 4 \leq \lambda_{n}$ are cusp forms and the continuous spectrum arises from the Eisenstein series $\bar{E}(z ; s)$ at $s=\frac{1}{2}+i R$ $(R \in \mathbb{R})$. The eigenvalue of $E(z ; s)$ is $\lambda=s(1-s)$, and if $s=\frac{1}{2}+i R$, then $E(z ; s)$ is real-valued after a rotation; we write $E^{*}\left(z ; \frac{1}{2}+i R\right) \in \mathbb{R}$ for this rotated function (cf. section 3.1).

Received by the editor September 21, 2006 and, in revised form, May 16, 2007.

2000 Mathematics Subject Classification. Primary 11F72; Secondary 11F03, 11F06, 11 Y 35.

Key words and phrases. Automorphic forms, spectral theory, computational number theory, Fourier coefficients, explicit machine computations, Phillips-Sarnak conjecture, $K$-Bessel function, Teichmüller space. 
When $\Gamma \backslash \mathcal{H}$ is of finite hyperbolic area it is known that the geodesic flow on the surface is ergodic Hop36. If, in addition, $\Gamma \backslash \mathcal{H}$ is compact the results of Shnirelman/Colin de Verdiere/Zelditch [Shn74, Col85, Zel87] lead to

$$
\lim _{n \rightarrow \infty} \frac{1}{\mu(F)} \int_{F}\left|\phi_{n}(z)\right|^{2} d \mu=\frac{1}{\mu(\Gamma \backslash \mathcal{H})}
$$

for every Jordan region $F \subseteq \Gamma \backslash \mathcal{H}$, with the possible exclusion of a set of $\lambda_{n}$ of density 0 . This result has been extended to non-compact surfaces such as the modular surface $P S L(2, \mathbb{Z}) \backslash \mathcal{H}$; cf. Zelditch [Zel91]. In [LS95] Luo and Sarnak showed that a corresponding result holds for the Eisenstein series on $P S L(2, \mathbb{Z}) \backslash \mathcal{H}$ without the exclusion of a subset of eigenvalues (this is called quantum unique equidistribution). Recently, Lindenstrauss Lin06 proved quantum unique ergodicity for arithmetic compact surfaces and a slightly weaker result in the non-compact case.

One may now proceed to consider even deeper lying questions about the statistical properties of the eigenstates. Bearing the above facts in mind, it is not unreasonable to suspect that the relative frequency measures

$$
\frac{\mu\left(\left\{z \in F ; \sigma^{-1} E^{*}\left(z ; \frac{1}{2}+i R\right) \in[a, b]\right\}\right)}{\mu(F)}
$$

over arbitrary Jordan regions $F \subseteq \Gamma \backslash \mathcal{H}$ should approach a standard $N(0,1)$ Gaussian distribution as $R \rightarrow \infty$ for a general surface $\Gamma \backslash \mathcal{H}$; cf. Ber77] and [HR92, $\S \S 6$, 7 (item 3)]. Here $\sigma$ is the standard deviation

$$
\sigma^{2}=\frac{1}{\mu(F)} \int_{F}\left|E^{*}\left(z ; \frac{1}{2}+i R\right)\right|^{2} d \mu .
$$

Several numerical experiments have previously been carried out which strongly support Gaussian distribution for cusp forms on $\Gamma \backslash \mathcal{H}$, with $\Gamma=P S L(2, \mathbb{Z})$ or $\Gamma$ a Hecke triangle group; cf. Hejhal and Rackner [HR92, Hej99 and, for $C M$-forms on congruence subgroups of $P S L(2, \mathbb{Z})$, Hejhal and Strömbergsson [HS01. Regarding the values of the Eisenstein series on the arithmetic surface $P S L(2, \mathbb{Z}) \backslash \mathcal{H}$, promising experimental results along with some heuristics are presented in [HR92].

This paper is mainly concerned with the Eisenstein series on non-arithmetic surfaces without any symmetries, i.e., in a setting where, in line with the PhillipsSarnak conjecture [PS85, there are expected to be (generically) no discrete eigenfunctions. The pursuit of quantum chaos must then depend entirely on the Eisenstein series!

In [Ave07] we worked with the group $\Gamma_{0}(5)$ and with groups $\Gamma$ in the Teichmüller space $T\left(\Gamma_{0}(5)\right)$ of $\Gamma_{0}(5)$. In particular, we examined cusp forms and Eisenstein series as the group $\Gamma$ is deformed in $T\left(\Gamma_{0}(5)\right)$, with the conjecture of Phillips and Sarnak in mind. In the present paper we make further use of the tools developed in Ave07. We will keep $\Gamma \in T\left(\Gamma_{0}(5)\right)$ fixed and compute the Eisenstein series in order to investigate statistical properties of its values and Fourier coefficients. More precisely, our two main topics are the value distribution of $E(z ; s)$ with $\operatorname{Im} s$ large, and statistics of Fourier coefficients of $E(z ; s)$. We consider $s$-values with $\operatorname{Re} s=1 / 2$ as well as ones having $\operatorname{Re} s>1 / 2$.

Our experimental results provide indications of Gaussian value distribution for the Eisenstein series. Indeed, when $\operatorname{Re} s=1 / 2$, our data suggests that the realvalued function $E^{*}(z ; s)$ has a limiting Gaussian distribution. When $\operatorname{Re} s>1 / 2$, $E(z ; s)$ is complex and we find reason to believe that $E(z ; s)$ approaches a complex 
Gaussian value distribution as $\operatorname{Im} s \rightarrow \infty$, at least on non-arithmetic groups and if we allow $\operatorname{Re} s \rightarrow 1 / 2$ at some rate. On the arithmetic group we cannot see resemblance of a complex Gaussian distribution when $\operatorname{Re} s>1 / 2$ due to a clustering phenomenon of Fourier coefficients that takes place for our values of $s$. The effect of letting $\operatorname{Im} s \rightarrow \infty$ here is not yet clear.

Regarding the Fourier coefficients we provide numerical evidence of a complex Gaussian value distribution in the non-arithmetic cases, when $\operatorname{Re} s=1 / 2$ or is near $1 / 2$ and we take the coefficients to be appropriately normalized.

\section{EISENSTEIN SERIES ON $\widetilde{\Gamma}_{a, r}$}

We will consider groups $\Gamma$ in the Teichmüller space of

$$
\Gamma_{0}(5)=\left\{\left(\begin{array}{ll}
a & b \\
c & d
\end{array}\right) \in P S L(2, \mathbb{Z}) ; c \equiv 0 \bmod 5\right\} .
$$

These groups have two cusps; this presents a computational difficulty which can be overcome by instead working with the one-cusp group

$$
\widetilde{\Gamma}_{0}(5)=\left\langle\Gamma_{0}(5), W_{0}\right\rangle,
$$

where $W_{0}$ is the Fricke involution $z \mapsto-\frac{1}{5 z}$. Functions $f(z)$ on $\Gamma_{0}(5) \backslash \mathcal{H}$ can be written $f(z)=f^{+}(z)+f^{-}(z)$ where $f^{ \pm}(z)$ are functions on $\widetilde{\Gamma}_{0}(5) \backslash \mathcal{H}$ with $f^{ \pm}\left(W_{0} z\right)= \pm f^{ \pm}(z)$. Moreover, if $\Delta f(z)+\lambda f(z)=0$, then also $\Delta f^{ \pm}(z)+\lambda f^{ \pm}(z)=$ 0 holds; cf. [Ave07, p. 4].

The Teichmüller space $T\left(\Gamma_{0}(5)\right)$ has dimension 2 (cf. [Ber72, p. 275]), and so there will be two real parameters to vary. We call these $a$ and $r$ and we will write $\Gamma_{a, r}$ and $\widetilde{\Gamma}_{a, r}$ (see the precise definition below) for the deformations of $\Gamma_{0}(5)$ and $\widetilde{\Gamma}_{0}(5)$. We remark that $(a, r)$ in fact provide real-analytic coordinates on $T\left(\Gamma_{0}(5)\right)$ (with respect to its standard complex analytic structure), at least for $|a|<0.05$ and $0.125<r<0.225$. A detailed proof of this is given in the Maple-file Ave04.

A discussion of the generators of the groups $\Gamma_{0}(5), \widetilde{\Gamma}_{0}(5), \Gamma_{a, r}$ and $\widetilde{\Gamma}_{a, r}$ can be found in [FL05, §3], where a more general case is considered, and in [Ave03] where we have worked out the details for our special case. (Note that the parameters in [FL05] correspond to ours as $a=b_{F L}$ and $r=1 / a_{F L}$.) Here we simply state the generators of $\Gamma_{a, r}$ and $\widetilde{\Gamma}_{a, r}$ in Table 1 .

$$
\begin{aligned}
& S= \pm\left(\begin{array}{ll}
1 & 1 \\
0 & 1
\end{array}\right), W= \pm\left(\begin{array}{cc}
a & -r-a^{2} \\
1 & -a
\end{array}\right), W S W= \pm\left(\begin{array}{cc}
1-\frac{a}{r} & \frac{a^{2}}{r} \\
-\frac{1}{r} & 1+\frac{a}{r}
\end{array}\right), \\
& E= \pm\left(\begin{array}{cc}
\frac{a-2 r-\sqrt{D}}{2} & \frac{(4 r-1) a+(2 a-1) \sqrt{D}}{2} \\
1 & \frac{-a+2 r+\sqrt{D}}{2}
\end{array}\right), \\
& W E W= \pm\left(\begin{array}{cc}
\frac{a+2 r+\sqrt{D}}{2} & \frac{(1-4 r) a-(2 a+1) \sqrt{D}}{2} \\
1 & \frac{-a-2 r-\sqrt{D}}{2}
\end{array}\right) \text {, where } D=4 r^{2}+a^{2}, \\
& \widetilde{\Gamma}_{a, r}=\langle S, E, W\rangle \quad\left(\text { relations } W^{2}=E^{2}=(S E W)^{2}=I\right), \\
& \Gamma_{a, r}=\langle S, E, W E W, W S W\rangle\left(\text { relations } E^{2}=(W E W)^{2}=(S E W)^{2}=I\right) .
\end{aligned}
$$

TABLE 1. The generators of $\Gamma_{a, r}$ and $\widetilde{\Gamma}_{a, r}$ (given as Möbius transformations; not all these matrices have determinant 1). 
We obtain the groups $\Gamma_{0}(5)$ and $\widetilde{\Gamma}_{0}(5)$ when $(a, r)=(0,1 / 5)$, i.e., $\Gamma_{0}(5)=\Gamma_{0, \frac{1}{5}}$ and $\widetilde{\Gamma}_{0}(5)=\widetilde{\Gamma}_{0, \frac{1}{5}}$.

On $\widetilde{\Gamma}_{a, r}$ one may define the Eisenstein series as

$$
E(z ; s)=\sum_{U \in\langle S\rangle \backslash \widetilde{\Gamma}_{a, r}}(\operatorname{Im} U z)^{s} \quad \text { for } \quad \operatorname{Re} s>1,
$$

and it has a meromorphic continuation to all $s \in \mathbb{C}[$ Hej83, p. 130]. Our numerical algorithm is a modified version of the algorithm for computations of Maass waveforms found in Hej99 and it is described in Ave07. It builds on the automorphy relation applied to a set of evenly spaced points along a closed horocycle $\operatorname{Im} z=Y$ (lying below the fundamental domain of the group), and it uses the Fourier series expansion of $E(z ; s)$ :

$$
E(z ; s)=y^{s}+\varphi(s) y^{1-s}+\sum_{1 \leq|m|<\infty} \varphi_{m}(s) y^{1 / 2} K_{s-\frac{1}{2}}(2 \pi|m| y) e^{2 \pi i m x},
$$

or, more precisely, a truncated version of it (for a suitable $M$ ):

$$
E(z ; s)=y^{s}+\varphi(s) y^{1-s}+\sum_{1 \leq|m| \leq M} \varphi_{m}(s) y^{1 / 2} K_{s-\frac{1}{2}}(2 \pi|m| y) e^{2 \pi i m x}+\varepsilon .
$$

Here $K_{s-1 / 2}(X)$ denotes the usual $K$-Bessel function multiplied by a factor $e^{\pi \operatorname{Im}(s) / 2}$; cf. Ave07, §4.3]. When the coefficients $\varphi_{-M}(s), \ldots, \varphi_{M}(s)$ and $\varphi(s)$ have been computed to sufficient accuracy we may obtain the higher coefficients $\varphi_{m}(s)$ with $|m|>M$ in exactly the same way as in [Hej99, §4].

Several tests of accuracy regarding the computations of $E(z ; s)$-values were discussed in Ave07, §4.5]. The data used in the present paper were mainly checked by repeating the computations of Fourier coefficients with a different parameter $Y$; cf. Hej99, §4]. The difference between the two results is denoted $\delta$. It gives us a measure of accuracy, but there is, a priori, no guarantee that it reflects the true accuracy. However, the arithmetic group $\widetilde{\Gamma}_{0}(5)$ offers an excellent possibility to check this: from Hej83, Ch. 11.4] one may deduce explicit formulas,

$$
\begin{gathered}
\varphi(s)=\frac{\pi^{\frac{1}{2}} \Gamma\left(s-\frac{1}{2}\right) \zeta(2 s-1)}{\Gamma(s) \zeta(2 s)} \cdot \frac{5^{1-s}+1}{5^{s}+1}, \\
e^{-\frac{\pi \operatorname{Im} s}{2} \varphi_{m}(s)}=\frac{2 \pi^{s}|m|^{s-\frac{1}{2}}}{\Gamma(s) \zeta(2 s)} \sum_{d \mid m} d^{1-2 s} \begin{cases}\frac{1}{5^{s}+1} & \text { if } 5 \nmid d \\
1 & \text { if } 5 \mid d .\end{cases}
\end{gathered}
$$

Our parameters $M$ and $Y$ were chosen with an aim to achieve 6 digit accuracy in the final data, and the above tests gave the following results: On $\widetilde{\Gamma}_{0}(5)$ we found that $99 \%$ of the coefficients with $|m|>M$ have at least 6 correct digits and the majority (about $90 \%$ ) have $6-8$ correct digits. About $95 \%$ of the $\delta$-values indicate the true accuracy or are at most one digit off. Thus, although $\delta$ is not an exact measure of accuracy it certainly gives a hint about our overall accuracy.

Coefficients with $|m|<M$ are used to compute $E(z ; s)$-values, and their accuracy is displayed in Table 2, Our groups here are:

$$
\Gamma_{1}=\widetilde{\Gamma}_{a, r} \text { with }(a, r)=(0.1,0.19), \quad \Gamma_{2}=\widetilde{\Gamma}_{a, r} \text { with }(a, r)=(0.15,0.17) .
$$

We stress that both $\Gamma_{1}$ and $\Gamma_{2}$ are non-arithmetic; for the results in Hel66] imply that if $\Gamma_{j}$ were arithmetic, then every $S L(2, \mathbb{R})$-matrix which represents an element 


\begin{tabular}{|c|c|ccc|}
\hline & $\operatorname{Im} s$ & $\operatorname{Re} s=0.5$ & $\operatorname{Re} s=0.501$ & $\operatorname{Re} s=0.55$ \\
\hline \hline \multirow{3}{*}{$\widetilde{\Gamma}_{0}(5)$} & 100 & 11 & 11 & 11 \\
& 500 & 9 & 9 & 10 \\
& 1000 & 7 & 8 & 10 \\
\hline \multirow{3}{*}{$\Gamma_{1}, \Gamma_{2}$} & 100 & 8 & 9 & 10 \\
& 500 & 7 & 8 & 10 \\
& 1000 & 6 & 7 & 9 \\
\hline
\end{tabular}

TABLE 2. The table displays the number of significant digits which apply for at least $99 \%$ of the coefficients with $|m|<M$. For $\widetilde{\Gamma}_{0}(5)$ these numbers are based on comparison with (6) and for $\Gamma_{1}, \Gamma_{2}$ they reflect the value of $\delta$.

in $\Gamma_{j}$ must have trace of the form $n \sqrt{e}$ for some integers $e, n$ with $e \geq 1$, and this fails e.g. for the element $W S \in \Gamma_{j}$ (which gives trace $\pm r^{-1 / 2}$ ).

For readers interested in the details of our implementation we remark that our computations of $E(z ; s)$ for $\operatorname{Im} s=1000$ had $M=1129$ in (44) and to compute $\varphi_{-M}(s), \ldots, \varphi_{M}(s)$ and $\varphi(s)$ we used $M_{0}=1206$ (notation as in Ave07, $\S 4.1$ ]; this corresponds to solving a $2412 \times 2412$ system of linear equations) and $Y$-values 0.145 and 0.135. Our choices of $M, M_{0}$ and $Y$ depend only on $\operatorname{Im} s$. When computing higher coefficients with the counterpart of Hej99, (16')] it is crucial to optimize $M_{0}$ and $Y$ in such a way that $K_{s-1 / 2}\left(2 \pi M_{0} Y\right)$ is small but not too small since then division by $K_{s-1 / 2}(2 \pi|m| Y)$ for $|m|>M_{0}$ will cause errors; cf. also [The05.

Finding one set of $\varphi_{-M}(s), \ldots, \varphi_{M}(s)$ for $\operatorname{Im} s=1000$ takes 5 hours on a 3199 $\mathrm{MHz}$ machine and uses 98.6 MB of memory. Computations of the value of $E(z ; s)$ at a single $z$ then takes $0.21 \mathrm{~s}$. In total, several weeks of computer time were spent on gathering data.

\section{VAlues AND COEFFICIENTS of $E(z ; s)$}

3.1. Symmetries in the complex plane. For the Eisenstein series on one-cusp groups we have the standard relations (see [Hej83, pp. 77, 130])

$$
E(z ; s)=\varphi(s) E(z ; 1-s), \quad \varphi(s) \varphi(1-s)=1 .
$$

Also $\overline{\varphi(s)}=\varphi(\bar{s})$ and $\overline{E(z ; s)}=E(z ; \bar{s})$. When $s=1 / 2+i R$ it follows that $\varphi(s)=e^{i \omega}$, for some $\omega \in \mathbb{R}$. Writing $E(z ; s)=r e^{i \theta}$ also in polar form we find that $r e^{i \theta}=e^{i \omega} r e^{-i \theta}$ and so, if $r \neq 0$, we must have $\theta=\omega / 2+\pi k$ with $k \in \mathbb{Z}$. This means that for $s=1 / 2+i R$ the values of the Eisenstein series are situated along a line with angle $\omega / 2$ to the real axis in the complex plane, and a rotation makes them real: We set $E^{*}(z ; s)=e^{-i \omega / 2} E(z ; s) \in \mathbb{R}$.

There are also consequences for the Fourier coefficients when $\operatorname{Re} s=1 / 2$. Since $E^{*}(z ; s) \in \mathbb{R}$ for all $z$ we find from (3) that the rotated coefficients $\psi_{m}(s)=$ $e^{-i \omega / 2} \varphi_{m}(s)$ satisfy $\psi_{-m}(s)=\overline{\psi_{m}(s)}$.

We will now explore this a bit further for the arithmetic group $\widetilde{\Gamma}_{0}(5)$. On $\widetilde{\Gamma}_{0}(5)$ the Eisenstein series is even in the sense that $\varphi_{-m}(s)=\varphi_{m}(s)$ with the effect that only terms with $\cos (2 \pi m x)$ are present in the Fourier expansion (3); cf. (6) and for more information e.g. Ave07, p. 8]. In this situation we have $\psi_{m}(s) \in \mathbb{R}$; cf. the first box of Figure 1 

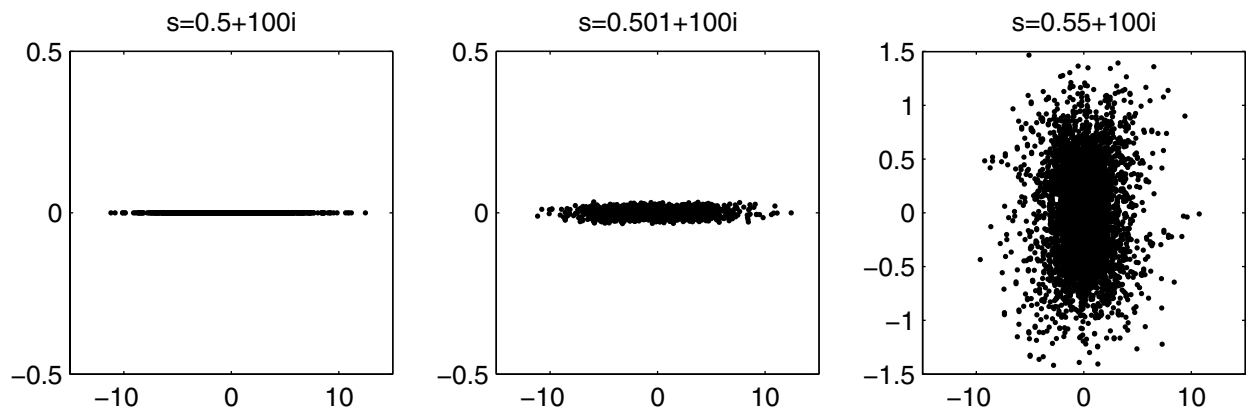

Figure 1. Plots of the first 10000 Fourier coefficients $e^{-i \omega_{1}} \varphi_{m}(s)$ of $E(z ; s)$ for $\widetilde{\Gamma}_{0}(5)$. Note the difference in scale of the real and imaginary axes.

When $\operatorname{Re} s \neq 1 / 2$ the coefficients and values for $E(z ; s)$ are no longer on a straight in line in the complex plane. However, as we let Re $s$ become slightly larger than $1 / 2$ we find the first 10000 coefficients clustering around a straight line; cf. Figure1. We may use the coefficient formula (6) to explain this.

For simplicity we look at a coefficient $\varphi_{m}(s)$ with $m \neq 5$ a prime. We let $s=1 / 2+\eta+i R$ with $\eta>0$ small. We may then write

$$
\begin{aligned}
& e^{-\frac{\pi \operatorname{Im} s}{2}} \varphi_{m}(s)=w\left(m^{\eta+i R}+m^{-\eta-i R}\right) \\
& =w\left(\left(m^{\eta}+m^{-\eta}\right) \cos (R \ln m)+i\left(m^{\eta}-m^{-\eta}\right) \sin (R \ln m)\right)
\end{aligned}
$$

with

$$
w=\frac{2 \pi^{s}}{\Gamma(s) \zeta(2 s)} \cdot \frac{1}{5^{s}+1} .
$$

Here $w \neq 0$ whenever $\eta>0$. It follows that these $\varphi_{m}(s)$ will lie near the line $e^{i \omega_{1}} \mathbb{R}$ with $\omega_{1}=\arg w$, and that the distance to $e^{i \omega_{1}} \mathbb{R}$ will depend on the size of $m^{\eta}$. This distance will be small as long as $m^{\eta}$ is close to 1 . (When $s=1 / 2+i R$ this agrees with our earlier notation: it is a simple exercise using functional equations to show that $\omega_{1}=\omega / 2$ for $s=1 / 2+i R$.) Treatment of other coefficients $\varphi_{m}(s)$ with $5 \nmid m$ is similar. Let $m \geq 1$. Instead of the second factor in (7) we have a sum of paired terms (this pairing of terms does not take place for $\varphi_{m}(s)$ with $5 \mid m$ ):

$$
\begin{aligned}
& e^{-\frac{\pi \operatorname{Im} s}{2}} \varphi_{m}(s)=w \sum_{\substack{d \mid m \\
1 \leq d \leq \sqrt{m}}}^{\prime}\left[\left(\left(\frac{m}{d^{2}}\right)^{\eta}+\left(\frac{m}{d^{2}}\right)^{-\eta}\right) \cos \left(R \log \frac{m}{d^{2}}\right)\right. \\
& \left.+i\left(\left(\frac{m}{d^{2}}\right)^{\eta}-\left(\frac{m}{d^{2}}\right)^{-\eta}\right) \sin \left(R \log \frac{m}{d^{2}}\right)\right] .
\end{aligned}
$$

The prime above the sum denotes that we count the $(d=\sqrt{m})$-term with a factor $1 / 2$ if it occurs. Thus all these coefficients will be near $e^{i \omega_{1}} \mathbb{R}$ if $\eta$ is sufficiently small (in a way depending on $m$ ).

This phenomenon is illustrated in Figures 1 and 2, Figure 1 includes the values of Re $s$ used for the statistical studies in this paper. In Figure 2 we keep $s$ fixed. The upper two plots include all coefficients up to $m=10000$ and $m=1000$, respectively. In the bottom plots we extract the $e^{-i \omega_{1}} \varphi_{m}(s)$ with $m$ prime. Here 

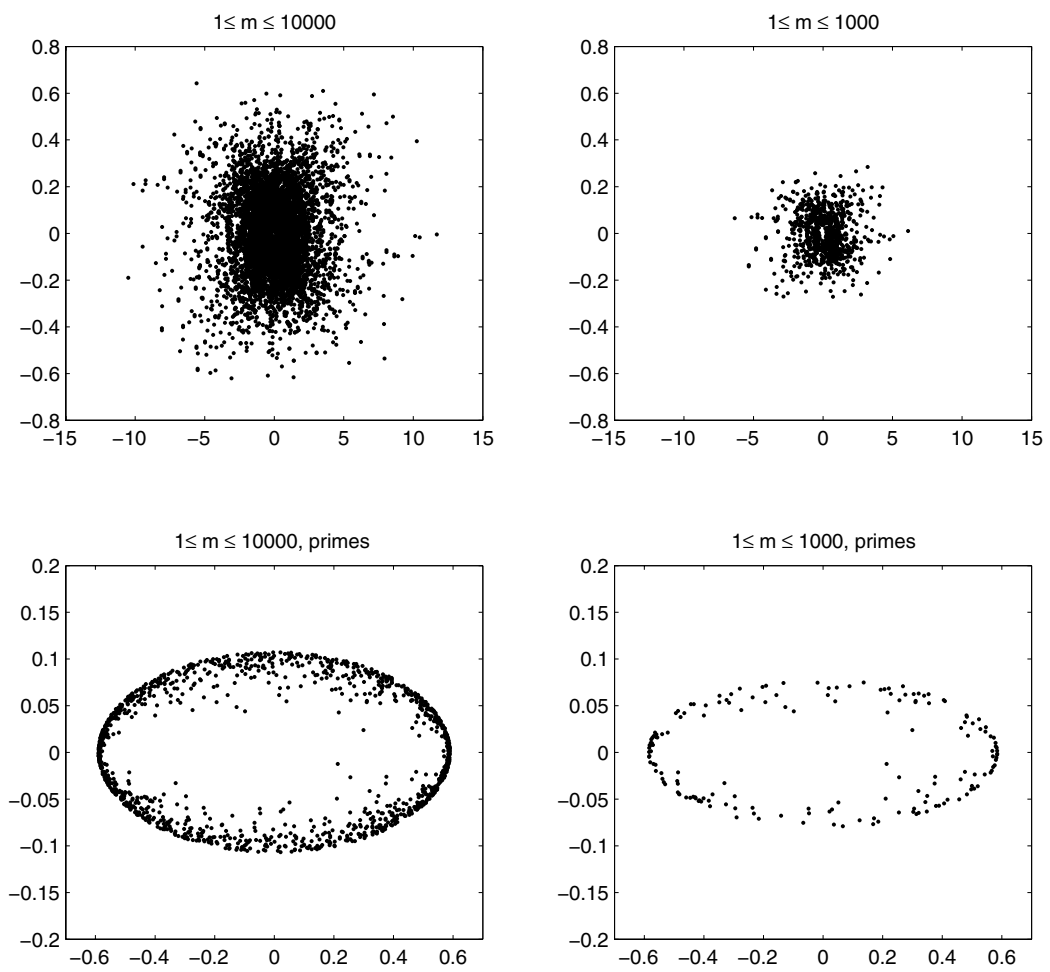

Figure 2. Fourier coefficients $e^{-i \omega_{1}} \varphi_{m}(s)$ of $E(z ; s)$ with $s=$ $0.52+100 i$ for $\widetilde{\Gamma}_{0}(5)$. The beautiful ellipse shape for prime coefficients is explained by (7).

the ellipse shape indicated by (7) is nicely visible and we also see clearly how the coefficients spread out from the real axis as we increase $m$.

3.2. Statistics for Fourier coefficients. In Hej99 Hejhal studied the statistical behavior of Fourier coefficients $d_{m}$ of cusp forms for Hecke triangle groups. The value distribution of the $d_{m}$ as $m \rightarrow \infty$ changes dramatically depending on whether the group is arithmetic or not. Prime coefficients $d_{p}$ on arithmetic triangle groups were observed to have the conjectured Sato-Tate distribution, but including all $d_{m}$ results in a Dirac delta-distribution because of multiplicativity. On non-arithmetic groups, where multiplicative relations are absent, Hejhal found experimental evidence of Gaussian distribution for the Fourier coefficients $d_{m}$.

The well-known Rankin-Selberg asymptotic formula holds for cusp forms (see BR99] for the proof of the error term for an arbitrary cofinite Fuchsian group):

$$
\sum_{1 \leq|m| \leq N}\left|d_{m}\right|^{2}=A_{1} N+O\left(N^{\frac{2}{3}+\varepsilon}\right),
$$

ensuring us that the limit value distribution of the coefficients (if it exists) has finite second moment. 
For the Eisenstein series on a cofinite Fuchsian group $\Gamma$, Strömbergsson Str05a proved the following asymptotic formulas. For $s=1 / 2+i R$ (regarding the factor $e^{\pi R}$; cf. the paragraph below (4)):

$$
e^{\pi R} \cdot \sum_{1 \leq|m| \leq N}\left|\varphi_{m}(s)\right|^{2}=c_{1} N \log N+c_{2} N+\operatorname{Re}\left(c_{3} N^{1+2 i R}\right)+O_{\Gamma, R}\left(N^{\frac{3}{4}+\varepsilon}\right)
$$

with

$$
\begin{aligned}
c_{1} & =\frac{16 \cosh (\pi R)}{\pi \mu(\Gamma \backslash \mathcal{H})}, \\
c_{2} & =\frac{8 \cosh (\pi R)}{\pi \mu(\Gamma \backslash \mathcal{H})}\left(-2+2 b-\frac{\varphi^{\prime}\left(\frac{1}{2}+i R\right)}{\varphi\left(\frac{1}{2}+i R\right)}-2 \operatorname{Re}\left(\frac{\Gamma^{\prime}\left(\frac{1}{2}+i R\right)}{\Gamma\left(\frac{1}{2}+i R\right)}\right)+2 \log (4 \pi)\right), \\
b & =\lim _{s \rightarrow 1}\left(\mu(\Gamma \backslash \mathcal{H}) \varphi(s)-(s-1)^{-1}\right), \\
c_{3} & =\frac{8 \Gamma(1+i R)}{\Gamma\left(\frac{1}{2}+2 i R\right) \Gamma\left(\frac{3}{2}+i R\right)} \cdot \varphi(1+2 i R) \varphi\left(\frac{1}{2}-i R\right) \cdot(2 \pi)^{2 i R},
\end{aligned}
$$

and for $s=\sigma+i R, \sigma>1 / 2, \sigma \neq 1, R \neq 0$ :

$$
e^{\pi R} \cdot \sum_{1 \leq|m| \leq N}\left|\varphi_{m}(s)\right|^{2} \approx d_{1} N^{2 \sigma}+d_{2} N^{2-2 \sigma}+\operatorname{Re}\left(d_{3} N^{1+2 i R}\right) \quad \text { as } \quad N \rightarrow \infty
$$

with

$$
\begin{aligned}
& d_{1}=\frac{8 \pi^{2 \sigma-\frac{1}{2}} \Gamma(2 \sigma)}{|\Gamma(s)|^{2} \Gamma\left(2 \sigma-\frac{1}{2}\right)} \cdot \frac{\varphi(2 \sigma)}{2 \sigma}, \\
& d_{2}=\frac{8 \pi^{\frac{3}{2}-2 \sigma} \Gamma(2-2 \sigma)}{|\Gamma(1-s)|^{2} \Gamma\left(\frac{3}{2}-2 \sigma\right)} \cdot \frac{\varphi(2-2 \sigma)}{2-2 \sigma}|\varphi(s)|^{2}, \\
& d_{3}=\frac{16 \pi^{\frac{1}{2}+2 i R} \Gamma(1+2 i R)}{\Gamma\left(\frac{1}{2}+2 i R\right) \Gamma(s) \Gamma(1-\bar{s})} \cdot \frac{\varphi(1+2 i R)}{1+2 i R} \cdot \overline{\varphi(s)}
\end{aligned}
$$

For the case of arithmetic groups this type of formula is well-known (cf. HR92, $\S 7])$.

To get an indication of the behavior of the true error in (8) and (9) and also as a check that all constants were evaluated correctly, we plotted the relevant differences in each of our cases. For example, Figure 3 illustrates (8) for $\Gamma_{1}$ and one of our typical choices of $s$. The corresponding pictures for our other cases are similar. Starting at the top, these plots are of

$$
\begin{aligned}
& e^{\pi R} \cdot \sum_{1 \leq|m| \leq N}\left|\varphi_{m}(s)\right|^{2}, \quad e^{\pi R} \cdot \sum_{1 \leq|m| \leq N}\left|\varphi_{m}(s)\right|^{2}-c_{1} N \log N, \\
& e^{\pi R} \cdot \sum_{1 \leq|m| \leq N}\left|\varphi_{m}(s)\right|^{2}-c_{1} N \log N-c_{2} N-\operatorname{Re}\left(c_{3} N^{1+2 i R}\right)
\end{aligned}
$$

with $N=10000$. Note how the magnitude decreases significantly as we subtract off more terms. The difference between the first two plots and the last one is striking; the last plot is much more "chaotic".

Just as for cusp forms, it is not known whether the coefficients of the Eisenstein series have a Gaussian value distribution. The above asymptotic formulas suggest that this question is not quite as natural as with cusp forms; we will not have finite second moments. But to be able to examine the value distribution of the first $N$ 

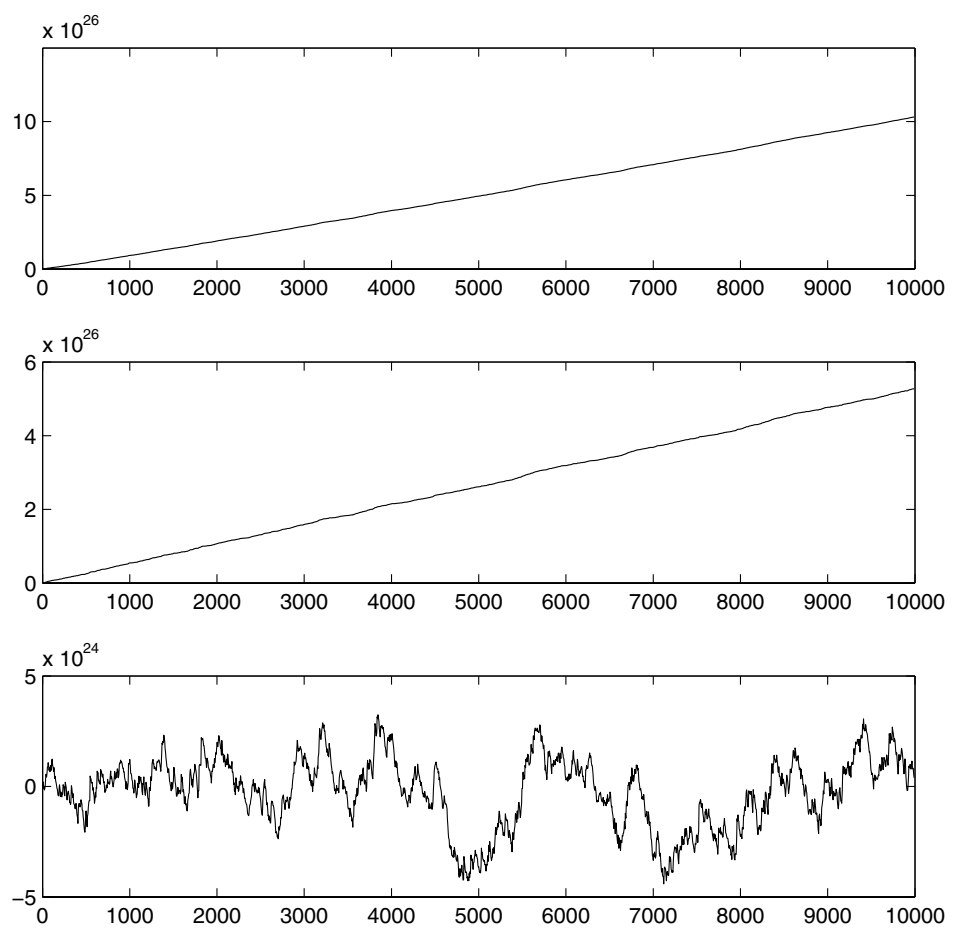

Figure 3 . The graphs of $e^{\pi R} \cdot \sum_{1 \leq|m| \leq N}\left|\varphi_{m}(s)\right|^{2}$ as a function of $N$ for $\Gamma_{1}, s=1 / 2+16 i$, with none, one, and three of the terms on the right hand side of (8) subtracted off, respectively. The bottom plot displays the chaotic behavior of the error term in this asymptotic formula.

coefficients $\varphi_{m}(s)$ we may construct something with second moment tending to a finite limit as $N \rightarrow \infty$ :

$$
\widetilde{\varphi}_{m}(s):=\frac{\varphi_{m}(s)}{\sqrt{\log N}} \quad \text { if } s=\frac{1}{2}+i R ; \quad \widetilde{\varphi}_{m}(s):=\frac{\varphi_{m}(s)}{N^{\eta}} \quad \text { if } s=\frac{1}{2}+\eta+i R .
$$

Note that these depend on $N$.

The results for non-arithmetic groups and $N=10000$ are shown in Tables 3 and 4. The groups $\Gamma_{1}$ and $\Gamma_{2}$ were defined in section 2 For $\operatorname{Re} s=1 / 2$ only $\widetilde{\varphi}_{m}(s)$ with $m$ positive are included because of the symmetry relations discussed in section 3.1. The following notation regarding standard deviation and moments are used if $\operatorname{Re} s>1 / 2$ (and corresponding formulas for $\operatorname{Re} s=1 / 2$ ):

$$
\sigma^{2}=\frac{1}{2 N-1} \sum_{1 \leq|m| \leq N}\left(\operatorname{Re} \widetilde{\varphi}_{m}(s)\right)^{2}, \quad I_{k}=1-\frac{\frac{1}{2 N} \sum_{1 \leq|m| \leq N}\left(\operatorname{Re} \widetilde{\varphi}_{m}(s)\right)^{k}}{\pi^{-\frac{1}{2}} 2^{\frac{k}{2}} \sigma^{k} \Gamma\left(\frac{k+1}{2}\right)}
$$

and similarly for the imaginary parts. Note that these formulas assume that the mean is 0 . (Thus $I_{k}=0$ if the numbers $\widetilde{\varphi}_{m}(s)$ are perfectly Gaussian distributed.) 
The value $\delta$ is the mean difference between the histogram points and the corresponding points on the Gaussian curve $(\sigma \sqrt{2 \pi})^{-1} \exp \left(-\frac{x^{2}}{2 \sigma^{2}}\right)$. We also give the minimum and maximum value of $\operatorname{Re} \widetilde{\varphi}_{m}(s)$ and $\operatorname{Im} \widetilde{\varphi}_{m}(s)$ for $|m| \leq 10000$.

\begin{tabular}{|c|cccccccc|}
\hline $\mathrm{s}$ & mean & $\sigma$ & $\delta$ & $\max$ & $\min$ & $I_{4}$ & $I_{6}$ & $I_{8}$ \\
\hline \hline $0.500+16 i$ & $-8 \mathrm{e}-03$ & 0.64 & $7 \mathrm{e}-03$ & 2.58 & -2.71 & $-1 \mathrm{e}-01$ & $-3 \mathrm{e}-01$ & $-4 \mathrm{e}-01$ \\
& $-3 \mathrm{e}-03$ & 0.65 & $1 \mathrm{e}-02$ & 2.64 & -2.87 & $-9 \mathrm{e}-02$ & $-2 \mathrm{e}-01$ & $-5 \mathrm{e}-01$ \\
\hline $0.501+16 i$ & $-2 \mathrm{e}-02$ & 1.91 & $2 \mathrm{e}-03$ & 7.63 & -9.29 & $-1 \mathrm{e}-01$ & $-3 \mathrm{e}-01$ & $-6 \mathrm{e}-01$ \\
& $-1 \mathrm{e}-02$ & 1.91 & $2 \mathrm{e}-03$ & 7.87 & -8.49 & $-1 \mathrm{e}-01$ & $-3 \mathrm{e}-01$ & $-5 \mathrm{e}-01$ \\
\hline $0.550+16 i$ & $-8 \mathrm{e}-03$ & 0.86 & $2 \mathrm{e}-03$ & 3.23 & -3.75 & $-2 \mathrm{e}-02$ & $-8 \mathrm{e}-02$ & $-2 \mathrm{e}-01$ \\
& $-3 \mathrm{e}-03$ & 0.86 & $3 \mathrm{e}-03$ & 3.68 & -3.54 & $-7 \mathrm{e}-03$ & $-2 \mathrm{e}-02$ & $-6 \mathrm{e}-02$ \\
\hline $0.500+100 i$ & $5 \mathrm{e}-03$ & 0.71 & $8 \mathrm{e}-03$ & 3.04 & -2.98 & $-1 \mathrm{e}-01$ & $-3 \mathrm{e}-01$ & $-6 \mathrm{e}-01$ \\
& $-4 \mathrm{e}-03$ & 0.71 & $7 \mathrm{e}-03$ & 2.67 & -2.75 & $-9 \mathrm{e}-02$ & $-2 \mathrm{e}-01$ & $-4 \mathrm{e}-01$ \\
\hline $0.501+100 i$ & $1 \mathrm{e}-02$ & 2.10 & $2 \mathrm{e}-03$ & 8.92 & -8.79 & $-1 \mathrm{e}-01$ & $-3 \mathrm{e}-01$ & $-6 \mathrm{e}-01$ \\
& $8 \mathrm{e}-04$ & 2.09 & $2 \mathrm{e}-03$ & 8.02 & -7.94 & $-9 \mathrm{e}-02$ & $-2 \mathrm{e}-01$ & $-4 \mathrm{e}-01$ \\
\hline $0.550+100 i$ & $5 \mathrm{e}-03$ & 0.77 & $5 \mathrm{e}-03$ & 3.16 & -3.20 & $-6 \mathrm{e}-02$ & $-2 \mathrm{e}-01$ & $-3 \mathrm{e}-01$ \\
& $7 \mathrm{e}-04$ & 0.77 & $3 \mathrm{e}-03$ & 3.32 & -3.45 & $-7 \mathrm{e}-02$ & $-2 \mathrm{e}-01$ & $-4 \mathrm{e}-01$ \\
\hline
\end{tabular}

TABle 3. Coefficient statistics for $\Gamma_{1}$; for each $s$ we give data for $\operatorname{Re} \widetilde{\varphi}_{m}(s)$ (in the first line) and $\operatorname{Im} \widetilde{\varphi}_{m}(s)$ (in the second line).

\begin{tabular}{|c|cccccccc|}
\hline$s$ & mean & $\sigma$ & $\delta$ & $\max$ & $\min$ & $I_{4}$ & $I_{6}$ & $I_{8}$ \\
\hline \hline $0.500+16 i$ & $6 \mathrm{e}-03$ & 0.73 & $4 \mathrm{e}-03$ & 3.27 & -2.92 & $1 \mathrm{e}-02$ & $2 \mathrm{e}-02$ & $-3 \mathrm{e}-02$ \\
& $-2 \mathrm{e}-03$ & 0.73 & $5 \mathrm{e}-03$ & 2.71 & -2.80 & $1 \mathrm{e}-02$ & $3 \mathrm{e}-02$ & $5 \mathrm{e}-02$ \\
\hline $0.501+16 i$ & $1 \mathrm{e}-02$ & 2.14 & $1 \mathrm{e}-03$ & 9.64 & -8.69 & $2 \mathrm{e}-02$ & $5 \mathrm{e}-02$ & $6 \mathrm{e}-02$ \\
& $5 \mathrm{e}-03$ & 2.16 & $9 \mathrm{e}-04$ & 8.05 & -8.28 & $2 \mathrm{e}-02$ & $4 \mathrm{e}-02$ & $9 \mathrm{e}-02$ \\
\hline $0.550+16 i$ & $4 \mathrm{e}-03$ & 0.88 & $2 \mathrm{e}-03$ & 3.57 & -3.49 & $1 \mathrm{e}-02$ & $3 \mathrm{e}-02$ & $6 \mathrm{e}-02$ \\
& $1 \mathrm{e}-03$ & 0.87 & $2 \mathrm{e}-03$ & 3.31 & -3.46 & $2 \mathrm{e}-03$ & $2 \mathrm{e}-02$ & $6 \mathrm{e}-02$ \\
\hline $0.500+100 i$ & $-4 \mathrm{e}-03$ & 0.64 & $5 \mathrm{e}-03$ & 2.53 & -2.53 & $-7 \mathrm{e}-04$ & $-2 \mathrm{e}-02$ & $-7 \mathrm{e}-02$ \\
& $-6 \mathrm{e}-03$ & 0.63 & $3 \mathrm{e}-03$ & 2.32 & -3.11 & $-2 \mathrm{e}-02$ & $-1 \mathrm{e}-01$ & $-4 \mathrm{e}-01$ \\
\hline $0.501+100 i$ & $-3 \mathrm{e}-03$ & 1.89 & $9 \mathrm{e}-04$ & 8.63 & -7.42 & $-1 \mathrm{e}-02$ & $-6 \mathrm{e}-02$ & $-1 \mathrm{e}-01$ \\
& $-2 \mathrm{e}-02$ & 1.88 & $1 \mathrm{e}-03$ & 6.91 & -9.20 & $-2 \mathrm{e}-02$ & $-9 \mathrm{e}-02$ & $-3 \mathrm{e}-01$ \\
\hline $0.550+100 i$ & $-7 \mathrm{e}-03$ & 0.78 & $3 \mathrm{e}-03$ & 3.32 & -3.00 & $4 \mathrm{e}-03$ & $-2 \mathrm{e}-02$ & $-8 \mathrm{e}-02$ \\
& $-4 \mathrm{e}-03$ & 0.78 & $2 \mathrm{e}-03$ & 3.22 & -3.24 & $3 \mathrm{e}-03$ & $2 \mathrm{e}-03$ & $-1 \mathrm{e}-02$ \\
\hline
\end{tabular}

TABLE 4. Coefficient statistics for $\Gamma_{2}$; for each $s$ we give data for $\operatorname{Re} \widetilde{\varphi}_{m}(s)$ (in the first line) and $\operatorname{Im} \widetilde{\varphi}_{m}(s)$ (in the second line).

The values $\delta$ in Tables 3 and 4 strongly indicate that the real and imaginary parts of the modified coefficients $\widetilde{\varphi}_{m}(s)$ have a Gaussian value distribution as $N \rightarrow \infty$. This is also supported by the $I_{4}$-values and to a lesser extent by the $I_{6^{-}}$and $I_{8^{-}}$ values; one should keep in mind that the higher moments are very sensitive even to quite small discrepancies versus the Gaussian curve.

The magnitude of the correlation coefficients between the real and imaginary parts range from 0.01 to 0.001 in these computations. It is reasonable to assume that in the limit $N \rightarrow \infty$, the true correlation is zero. Note also that the real and imaginary parts have nearly the same $\sigma$. Thus two necessary conditions for $\widetilde{\varphi}_{m}(s)$ to have a complex Gaussian distribution seem to be fulfilled. In fact, assuming the limit correlation to be zero, it is sufficient to show that every linear combination 
$\cos (\theta) \operatorname{Re} \widetilde{\varphi}_{m}(s)+\sin (\theta) \operatorname{Im} \widetilde{\varphi}_{m}(s)$ is Gaussian with the same $\sigma$; cf. e.g. Gut95, Ch. 5]. Experiments were carried out for 6 such linear combinations for each $s$ value in Tables 3 and 4 . In Table 5 we show the results for $\Gamma_{1}, s=0.55+16 i$; the other cases are of similar quality. Clearly the agreement with the Gaussian is the same in all 6 directions and $\sigma$ is stable. Therefore it seems reasonable to conjecture that $\widetilde{\varphi}_{m}(s)$ has a complex Gaussian distribution for Re $s$ near $1 / 2$.

\begin{tabular}{|c|cccccccc|}
\hline$\theta$ & $\operatorname{mean}$ & $\sigma$ & $\delta$ & $\max$ & $\min$ & $I_{4}$ & $I_{6}$ & $I_{8}$ \\
\hline \hline 0 & $-7 \mathrm{e}-03$ & 0.86 & $2 \mathrm{e}-03$ & 3.23 & -3.75 & $-2 \mathrm{e}-02$ & $-8 \mathrm{e}-02$ & $-1 \mathrm{e}-01$ \\
$\pi / 2$ & $-2 \mathrm{e}-03$ & 0.86 & $3 \mathrm{e}-03$ & 3.68 & -3.54 & $-7 \mathrm{e}-03$ & $-2 \mathrm{e}-02$ & $-6 \mathrm{e}-02$ \\
0.5 & $-7 \mathrm{e}-03$ & 0.86 & $2 \mathrm{e}-03$ & 3.35 & -3.76 & $-2 \mathrm{e}-02$ & $-6 \mathrm{e}-02$ & $-1 \mathrm{e}-01$ \\
0.8 & $-7 \mathrm{e}-03$ & 0.86 & $2 \mathrm{e}-03$ & 3.48 & -3.56 & $-1 \mathrm{e}-02$ & $-5 \mathrm{e}-02$ & $-1 \mathrm{e}-01$ \\
2.0 & $6 \mathrm{e}-04$ & 0.87 & $3 \mathrm{e}-03$ & 4.00 & -3.13 & $-4 \mathrm{e}-03$ & $-1 \mathrm{e}-02$ & $-5 \mathrm{e}-02$ \\
4.0 & $7 \mathrm{e}-03$ & 0.86 & $2 \mathrm{e}-03$ & 3.61 & -3.56 & $-1 \mathrm{e}-02$ & $-4 \mathrm{e}-02$ & $-1 \mathrm{e}-01$ \\
\hline
\end{tabular}

TABLE 5. The consistency of Gaussian agreement for arbitrary linear combinations of real and imaginary parts supports our hypothesis about complex Gaussian distribution. As an example we include statistics for $\cos (\theta) \operatorname{Re} \widetilde{\varphi}_{m}(s)+\sin (\theta) \operatorname{Im} \widetilde{\varphi}_{m}(s), \Gamma_{1}, s=0.55+$ $16 i$.

On the non-arithmetic groups we have also computed correlation coefficients $\rho_{q}$ for shifted Fourier coefficients $\varphi_{m}(s), \varphi_{m+q}(s)$, with $q=1, \ldots, 5$ :

$$
\rho_{q}=\frac{\sum_{m=1}^{N} \varphi_{m}(s) \varphi_{m+q}(s)}{\sum_{m=1}^{N}\left|\varphi_{m}(s)\right|^{2}},
$$

with $N$ near 10000 ; see Table 6. Averages of this type are important in many theoretical questions; cf. e.g. [LS95, Sar01, LS03, LS04]. The $\rho_{q}$-values in Table 6] suggest that $\varphi_{m}(s)$ and $\varphi_{m+q}(s)$ are uncorrelated. Indeed, if the $\varphi_{m}(s)$ 's are replaced by independent Gaussian distributed random numbers, the resulting values of $\rho_{q}$ are found to be of the same order of magnitude as in Table 6 .

\begin{tabular}{|c|l|ccccc|}
\hline & $s$ & $q=1$ & $q=2$ & $q=3$ & $q=4$ & $q=5$ \\
\hline \hline \multirow{5}{*}{$\Gamma_{1}$} & $0.500+16 i$ & 0.00965 & 0.00981 & 0.01687 & 0.01974 & 0.00802 \\
& $0.501+16 i$ & 0.00986 & 0.00971 & 0.01692 & 0.01979 & 0.00803 \\
& $0.550+16 i$ & 0.01435 & 0.00647 & 0.01182 & 0.01508 & 0.00797 \\
& $0.500+100 i$ & 0.00784 & 0.00844 & 0.01472 & 0.01329 & 0.01235 \\
& $0.501+100 i$ & 0.00668 & 0.00863 & 0.01477 & 0.01292 & 0.01283 \\
& $0.550+100 i$ & 0.00952 & 0.01681 & 0.00830 & 0.00280 & 0.00858 \\
\hline \multirow{5}{*}{$\Gamma_{2}$} & $0.500+16 i$ & 0.00796 & 0.00213 & 0.01422 & 0.00703 & 0.01311 \\
& $0.501+16 i$ & 0.00789 & 0.00211 & 0.01450 & 0.00713 & 0.01324 \\
& $0.550+16 i$ & 0.01563 & 0.00228 & 0.02679 & 0.02374 & 0.01349 \\
& $0.500+100 i$ & 0.00230 & 0.00897 & 0.00960 & 0.00629 & 0.00897 \\
& $0.501+100 i$ & 0.00243 & 0.00858 & 0.00976 & 0.00641 & 0.00896 \\
& $0.550+100 i$ & 0.00583 & 0.00588 & 0.00575 & 0.00887 & 0.00554 \\
\hline
\end{tabular}

TABLE 6. Absolute values of the correlation coefficient $\rho_{q}$. 
As in Hej99 the picture becomes a different one for the arithmetic group. In fact, for $\operatorname{Re} s=1 / 2$, the value distribution for the coefficients $\widetilde{\varphi}_{m}(s)$ does not converge to a Gaussian distribution, at least not as far as moment convergence is concerned; cf. [Str05b]. Indeed, the fourth moment $\frac{1}{N} \sum_{1 \leq|m| \leq N}\left(\widetilde{\varphi}_{m}(s)\right)^{4}$ already diverges as $N \rightarrow \infty$. The proof uses a mimic of MS83] in the much simpler case of the divisor function in (6) to show that $\sum_{m=1}^{N}\left(\varphi_{m}(s)\right)^{4} \sim c N(\log N)^{6}$, with $c>0$, as $N \rightarrow \infty$.

3.3. Values of $E(z ; s)$. Recall that we expect $E^{*}(z ; 1 / 2+i R)$ to have a Gaussian value distribution as $R \rightarrow \infty$ (cf. section [1), i.e., we should have, for any $-\infty<$ $a<b<\infty$ and any compact Jordan subregion $F$ of $\Gamma \backslash \mathcal{H}$,

$$
\lim _{R \rightarrow \infty} \frac{\frac{1}{\mu(F)} \int_{F} \chi_{a b}\left(\sigma^{-1} E^{*}\left(z ; \frac{1}{2}+i R\right)\right) d \mu}{\frac{1}{\sqrt{2 \pi}} \int_{a}^{b} e^{-u^{2} / 2} d u}=1,
$$

where $\sigma(=\sigma(R))$ is the standard deviation $\sigma^{2}=\frac{1}{\mu(F)} \int_{F}\left(E^{*}(z ; 1 / 2+i R)\right)^{2} d \mu$, and $\chi_{a b}$ is the characteristic function of the interval $[a, b]$.

For $\operatorname{Re} s \neq 1 / 2, E(z ; s)$ itself does not take part in the spectral decomposition of $L^{2}(\Gamma \backslash \mathcal{H})$, but the value distribution for such $s$-values is also interesting. For example, one may ask if $E(z ; s)$ has a limit distribution for each fixed $\operatorname{Re} s$ as $\operatorname{Im} s \rightarrow \infty$.

We note that for $\operatorname{Re} s>1$ it follows from (2) that we do not have a Gaussian limit distribution in general. Indeed, if $\Gamma$ is any one-cusp group with a normalized cusp at $\infty, c>0$ is an arbitrarily small constant, and $F$ is any compact region with $\sup _{U \in \Gamma \backslash\langle S\rangle, z \in F} \operatorname{Im} U z<\inf _{z \in F} \operatorname{Im} z$, then whenever $\operatorname{Re} s$ is sufficiently large we have from (2), for all $z \in F: E(z ; s) \approx y^{s}$, with an absolute error less than $c y^{\operatorname{Re} s}$. This clearly makes it impossible for Gaussian distribution to hold as $\operatorname{Im} s \rightarrow \infty$. The same fact (i.e., $\left.\left|E(z ; s)-y^{s}\right|<c y^{\operatorname{Re} s}\right)$ is also true for any given $\operatorname{Re} s>1$, as long as the compact region $F$ is chosen sufficiently high up in the fundamental region (cf. Hej83, p. 56 (Prop. 8.1(e))], which is easily extended to fixed Re $s>1$ and arbitrary $\operatorname{Im} s$ ). In fact, it seems possible that by a more refined analysis one might be able to rule out, for any fixed $\operatorname{Re} s>1$ and $F$, the possibility of a Gaussian limit distribution as $\operatorname{Im} s \rightarrow \infty$.

Perhaps these facts hint that we should not expect to see a Gaussian limit distribution for any fixed $\operatorname{Re} s>1 / 2$ as $\operatorname{Im} s \rightarrow \infty$. However, it might be reasonable, for Re $s$ near $1 / 2$, to expect the limit distribution to resemble a Gaussian distribution.

In our numerical experiments we keep Re $s$ close to $1 / 2$. We include the arithmetic group $\widetilde{\Gamma}_{0}(5)$, as well as the non-arithmetic ones, $\Gamma_{1}$ and $\Gamma_{2}$ (cf. section 3.2). Recall that $\operatorname{Re} s=1 / 2$. We defined $E^{*}(z ; s)=e^{-i \omega / 2} E(z ; s) \in \mathbb{R}$ when $\operatorname{Re} s \neq 1 / 2$ we set $E^{*}(z ; s)=e^{-i \omega_{1}} E(z ; s)$ on $\widetilde{\Gamma}_{0}(5)$ and $E^{*}(z ; s)=E(z ; s)$ otherwise (cf. section 3.1 for explanations of $\omega$ and $\left.\omega_{1}\right)$.

Tables 7 8, 9 summarize our findings. We have used $F=[0,0.4] \times[1,1.4]$ and computed values of $E(z ; s)$ over a $1200 \times 1200$ grid for $\operatorname{Im} s \leq 500$ and a $2500 \times 2500$ grid for $\operatorname{Im} s=1000$. Histograms were then made by throwing hyperbolic area into 20 buckets according to the local size of the real and imaginary parts of $E^{*}(z ; s)$.

\footnotetext{
${ }^{1}$ In our case, some care is needed in the treatment of the special $p=5$ factor in the Euler products.
} 
The notation is the same as in section 3.2 except that now we have

$$
\sigma^{2}=\frac{1}{\mu(F)} \int_{F}\left|\operatorname{Re} E^{*}(z ; s)\right|^{2} d \mu, \quad I_{k}=1-\frac{\frac{1}{\mu(F)} \int_{F}\left|\operatorname{Re} E^{*}(z ; s)\right|^{k} d \mu}{\pi^{-\frac{1}{2}} 2^{\frac{k}{2}} \sigma^{k} \Gamma\left(\frac{k+1}{2}\right)},
$$

and similarly for $\operatorname{Im} E^{*}(z ; s)$. The value $c c$ is the correlation coefficient between real and imaginary parts. The integrals giving $\sigma$ and $I_{k}$ in (12) were computed as Riemann sums.

\begin{tabular}{|l|cccccc|}
\hline \multicolumn{1}{|c|}{$s$} & mean & $\sigma$ & $\delta$ & $I_{4}$ & $I_{6}$ & $I_{8}$ \\
\hline \hline $0.500+100 i$ & $1 \mathrm{e}-01$ & 1.93 & $2 \mathrm{e}-02$ & $2 \mathrm{e}-01$ & $5 \mathrm{e}-01$ & $7 \mathrm{e}-01$ \\
$0.500+500 i$ & $-1 \mathrm{e}-02$ & 2.34 & $3 \mathrm{e}-03$ & $1 \mathrm{e}-01$ & $2 \mathrm{e}-01$ & $4 \mathrm{e}-01$ \\
$0.500+1000 i$ & $-1 \mathrm{e}-02$ & 2.55 & $2 \mathrm{e}-03$ & $7 \mathrm{e}-02$ & $2 \mathrm{e}-01$ & $3 \mathrm{e}-01$ \\
\hline $0.501+100 i$ & $1 \mathrm{e}-01$ & 1.92 & $2 \mathrm{e}-02$ & $2 \mathrm{e}-01$ & $5 \mathrm{e}-01$ & $7 \mathrm{e}-01$ \\
$0.501+500 i$ & $-1 \mathrm{e}-02$ & 2.33 & $3 \mathrm{e}-03$ & $1 \mathrm{e}-01$ & $2 \mathrm{e}-01$ & $4 \mathrm{e}-01$ \\
$0.501+1000 i$ & $-1 \mathrm{e}-02$ & 2.53 & $2 \mathrm{e}-03$ & $7 \mathrm{e}-02$ & $2 \mathrm{e}-01$ & $3 \mathrm{e}-01$ \\
\hline $0.550+100 i$ & $1 \mathrm{e}-01$ & 1.65 & $2 \mathrm{e}-02$ & $2 \mathrm{e}-01$ & $5 \mathrm{e}-01$ & $7 \mathrm{e}-01$ \\
$0.550+500 i$ & $-9 \mathrm{e}-03$ & 1.75 & $7 \mathrm{e}-03$ & $1 \mathrm{e}-01$ & $3 \mathrm{e}-01$ & $4 \mathrm{e}-01$ \\
$0.550+1000 i$ & $-9 \mathrm{e}-03$ & 1.80 & $3 \mathrm{e}-03$ & $8 \mathrm{e}-02$ & $2 \mathrm{e}-01$ & $4 \mathrm{e}-01$ \\
\hline
\end{tabular}

TABLE 7. Value statistics of $\operatorname{Re} E^{*}(z ; s)$ for $\widetilde{\Gamma}_{0}(5)$.

\begin{tabular}{|l|ccccccc|}
\hline \multicolumn{1}{|c|}{$\mathrm{s}$} & $c c$ & mean & $\sigma$ & $\delta$ & $I_{4}$ & $I_{6}$ & $I_{8}$ \\
\hline \hline $0.500+100 i$ & & $8 \mathrm{e}-02$ & 2.92 & $4 \mathrm{e}-03$ & $1 \mathrm{e}-01$ & $3 \mathrm{e}-01$ & $5 \mathrm{e}-01$ \\
$0.500+500 i$ & & $1 \mathrm{e}-02$ & 12.41 & $1 \mathrm{e}-04$ & $9 \mathrm{e}-03$ & $3 \mathrm{e}-02$ & $5 \mathrm{e}-02$ \\
$0.500+1000 i$ & & $-6 \mathrm{e}-03$ & 27.03 & $3 \mathrm{e}-05$ & $5 \mathrm{e}-03$ & $2 \mathrm{e}-02$ & $4 \mathrm{e}-02$ \\
\hline \hline $0.501+100 i$ & -0.08 & $8 \mathrm{e}-02$ & 2.86 & $4 \mathrm{e}-03$ & $1 \mathrm{e}-01$ & $3 \mathrm{e}-01$ & $5 \mathrm{e}-01$ \\
& & $3 \mathrm{e}-03$ & 0.26 & $5 \mathrm{e}-02$ & $-4 \mathrm{e}-02$ & $-9 \mathrm{e}-02$ & $-7 \mathrm{e}-02$ \\
\hline $0.501+500 i$ & 0.02 & $6 \mathrm{e}-03$ & 8.05 & $2 \mathrm{e}-04$ & $8 \mathrm{e}-03$ & $3 \mathrm{e}-02$ & $5 \mathrm{e}-02$ \\
& & $-2 \mathrm{e}-03$ & 3.03 & $5 \mathrm{e}-04$ & $4 \mathrm{e}-03$ & $1 \mathrm{e}-02$ & $3 \mathrm{e}-02$ \\
\hline $0.501+1000 i$ & -0.38 & $-4 \mathrm{e}-03$ & 8.19 & $2 \mathrm{e}-04$ & $3 \mathrm{e}-03$ & $2 \mathrm{e}-02$ & $5 \mathrm{e}-02$ \\
& & $4 \mathrm{e}-03$ & 8.32 & $1 \mathrm{e}-04$ & $-2 \mathrm{e}-03$ & $-3 \mathrm{e}-02$ & $-9 \mathrm{e}-02$ \\
\hline \hline $0.550+100 i$ & 0.36 & $5 \mathrm{e}-02$ & 1.60 & $1 \mathrm{e}-02$ & $2 \mathrm{e}-01$ & $3 \mathrm{e}-01$ & $5 \mathrm{e}-01$ \\
& & $3 \mathrm{e}-02$ & 0.77 & $2 \mathrm{e}-02$ & $1 \mathrm{e}-01$ & $3 \mathrm{e}-01$ & $4 \mathrm{e}-01$ \\
\hline $0.550+500 i$ & 0.25 & $-6 \mathrm{e}-03$ & 1.51 & $3 \mathrm{e}-03$ & $7 \mathrm{e}-02$ & $2 \mathrm{e}-01$ & $3 \mathrm{e}-01$ \\
& & $2 \mathrm{e}-03$ & 1.25 & $3 \mathrm{e}-03$ & $6 \mathrm{e}-02$ & $2 \mathrm{e}-01$ & $3 \mathrm{e}-01$ \\
\hline $0.550+1000 i$ & -0.04 & $-2 \mathrm{e}-03$ & 1.48 & $3 \mathrm{e}-03$ & $6 \mathrm{e}-02$ & $1 \mathrm{e}-01$ & $2 \mathrm{e}-01$ \\
& & $6 \mathrm{e}-03$ & 1.35 & $3 \mathrm{e}-03$ & $6 \mathrm{e}-02$ & $1 \mathrm{e}-01$ & $2 \mathrm{e}-01$ \\
\hline
\end{tabular}

TABle 8. Value statistics of $E^{*}(z ; s)$ for $\Gamma_{1}$. Data for non-zero real and imaginary parts are included in that order.

Recall that for the arithmetic group $\widetilde{\Gamma}_{0}(5)$ and $\operatorname{Re} s$ near $1 / 2$ we found that the majority of the coefficients $\psi_{m}(s)=e^{-i \omega_{1}} \varphi_{m}(s)$ with $m$ not too large lie close to the real line; cf. e.g. Figure 1. The effect of this on the values of $E^{*}(z ; s)=e^{-i \omega_{1}} E(z ; s)$ is easy to explain heuristically (and to check numerically) if we consider its Fourier expansion:

$$
E^{*}(z ; s)=e^{-i \omega_{1}}\left(y^{s}+\varphi(s) y^{1-s}\right)+2 \sum_{m=1}^{\infty} \psi_{m}(s) y^{1 / 2} K_{s-\frac{1}{2}}(2 \pi|m| y) \cos (2 \pi m x) .
$$

As long as $\operatorname{Im} s$ is kept moderate only $\psi_{m}(s)$ with $|m|$ small are relevant in this sum (think of the truncated (4)!). The imaginary parts of the majority of such $\psi_{m}(s)$ 


\begin{tabular}{|l|ccccccc|}
\hline \multicolumn{1}{|c|}{$s$} & $c c$ & mean & $\sigma$ & $\delta$ & $I_{4}$ & $I_{6}$ & $I_{8}$ \\
\hline \hline $0.500+100 i$ & & $7 \mathrm{e}-02$ & 3.21 & $4 \mathrm{e}-03$ & $7 \mathrm{e}-02$ & $2 \mathrm{e}-01$ & $3 \mathrm{e}-01$ \\
$0.500+500 i$ & & $-6 \mathrm{e}-03$ & 9.75 & $2 \mathrm{e}-04$ & $3 \mathrm{e}-02$ & $9 \mathrm{e}-02$ & $2 \mathrm{e}-01$ \\
$0.500+1000 i$ & & $5 \mathrm{e}-03$ & 12.86 & $1 \mathrm{e}-04$ & $7 \mathrm{e}-03$ & $1 \mathrm{e}-02$ & $1 \mathrm{e}-02$ \\
\hline \hline $0.501+100 i$ & 0.99 & $8 \mathrm{e}-03$ & 0.40 & $3 \mathrm{e}-02$ & $6 \mathrm{e}-02$ & $1 \mathrm{e}-01$ & $3 \mathrm{e}-01$ \\
& & $7 \mathrm{e}-02$ & 3.14 & $4 \mathrm{e}-03$ & $7 \mathrm{e}-02$ & $2 \mathrm{e}-01$ & $3 \mathrm{e}-01$ \\
\hline $0.501+500 i$ & 0.84 & $-7 \mathrm{e}-03$ & 7.83 & $3 \mathrm{e}-04$ & $3 \mathrm{e}-02$ & $9 \mathrm{e}-02$ & $2 \mathrm{e}-01$ \\
& & $2 \mathrm{e}-03$ & 2.69 & $7 \mathrm{e}-04$ & $5 \mathrm{e}-03$ & $8 \mathrm{e}-03$ & $-2 \mathrm{e}-03$ \\
\hline $0.501+1000 i$ & 0.74 & $3 \mathrm{e}-03$ & 9.61 & $1 \mathrm{e}-04$ & $7 \mathrm{e}-03$ & $1 \mathrm{e}-02$ & $1 \mathrm{e}-02$ \\
& & $4 \mathrm{e}-03$ & 3.29 & $4 \mathrm{e}-04$ & $8 \mathrm{e}-03$ & $2 \mathrm{e}-02$ & $5 \mathrm{e}-02$ \\
\hline \hline $0.550+100 i$ & 0.04 & $1 \mathrm{e}-02$ & 0.69 & $1 \mathrm{e}-02$ & $-1 \mathrm{e}-01$ & $-3 \mathrm{e}-01$ & $-4 \mathrm{e}-01$ \\
& & $6 \mathrm{e}-02$ & 1.57 & $7 \mathrm{e}-03$ & $1 \mathrm{e}-01$ & $3 \mathrm{e}-01$ & $5 \mathrm{e}-01$ \\
\hline $0.550+500 i$ & -0.05 & $-8 \mathrm{e}-03$ & 1.43 & $3 \mathrm{e}-03$ & $6 \mathrm{e}-02$ & $1 \mathrm{e}-01$ & $2 \mathrm{e}-01$ \\
& & $5 \mathrm{e}-03$ & 1.49 & $2 \mathrm{e}-03$ & $6 \mathrm{e}-02$ & $2 \mathrm{e}-01$ & $2 \mathrm{e}-01$ \\
\hline $0.550+1000 i$ & 0.03 & $-1 \mathrm{e}-03$ & 1.45 & $2 \mathrm{e}-03$ & $5 \mathrm{e}-02$ & $1 \mathrm{e}-01$ & $2 \mathrm{e}-01$ \\
& & $7 \mathrm{e}-03$ & 1.38 & $3 \mathrm{e}-03$ & $6 \mathrm{e}-02$ & $2 \mathrm{e}-01$ & $3 \mathrm{e}-01$ \\
\hline
\end{tabular}

TABle 9. Value statistics of $E^{*}(z ; s)$ for $\Gamma_{2}$. Data for non-zero real and imaginary parts are included in that order.

are small compared to their real parts when $\operatorname{Re} s$ is near $1 / 2$. The same holds for $K_{s-1 / 2}(2 \pi|m| y)$. It follows that the imaginary part of the sum over $m$ above is small compared to its real part, the consequence being that the constant term $\operatorname{Im}\left(e^{-i \omega_{1}}\left(y^{s}+\varphi(s) y^{1-s}\right)\right)$ is dominating in size. Without the comparable impact of higher modes we cannot hope to see resemblance of a Gaussian distribution for

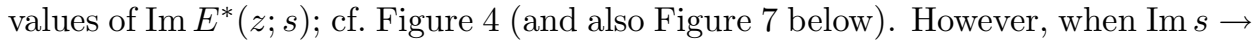
$\infty$ large $m$ come into play, there is still hope for a Gaussian limit distribution.

Resemblance to a Gaussian distribution can only be expected when the window $F$ is large compared to $\tau=\pi / \operatorname{Im} s$, which is related to the de Broglie wavelength. For $\operatorname{Im} s=100,500,1000$ the window $F$ corresponds to $13 \tau, 64 \tau$ and $127 \tau$, respectively. For $\operatorname{Im} s=100$ this is not enough. As $\operatorname{Im} s$ grows, however, we see a good improvement in $\delta$ in all our cases (except the case $\operatorname{Im} E^{*}(z ; s), \widetilde{\Gamma}_{0}(5), \operatorname{Re} s>1 / 2$ described above), as well as an overall improvement in the $I_{k}$-values, $k=4,6,8$; cf. also Figures 5 and 6 where we show histograms for $\widetilde{\Gamma}_{0}(5)$ and $\Gamma_{1}$ with $\operatorname{Re} s=1 / 2$.

Comparing Table 7 with Tables 8 and 9, and Figure 5 with Figure 6, we find that the quality of the Gaussian fit is much better for $\Gamma_{1}$ and $\Gamma_{2}$ (these two being about the same, quality-wise) than for $\widetilde{\Gamma}_{0}(5)$. Note especially that the improvement for the non-arithmetic cases as $\operatorname{Im} s$ grows is stronger. It is perhaps natural to expect a better Gaussian fit for non-arithmetic groups where no coefficient formulas are available.

In the cases where our experiments indicate that both $\operatorname{Re} E^{*}(z ; s)$ and $\operatorname{Im} E^{*}(z ; s)$ resemble a Gaussian value distributions as $\operatorname{Im} s \rightarrow \infty$ the question arises whether $E(z ; s)$ resembles a complex Gaussian distribution. Looking at the values of $c c$ and $\sigma$ in Tables 8 and 9 one might suspect that this is indeed the case and that the convergence is slower when $\operatorname{Re} s$ is close to $1 / 2$. The best candidates for testing complex Gaussian seem to be $s=0.55+1000 i$ for $\Gamma_{1}$ and $\Gamma_{2}$. We have computed the standard deviation of random linear combinations of $\operatorname{Re} E(z ; s)$ and $\operatorname{Im} E(z ; s)$ in the same way as in section 3.2, and the results indeed indicate that $E(z ; s)$ has a complex Gaussian limit distribution as $\operatorname{Im} s \rightarrow \infty$ (where we perhaps might allow $\operatorname{Re} s \rightarrow 1 / 2$ not too fast); cf. Table 10 and recall that $E(z ; s)=E^{*}(z ; s)$ here. 

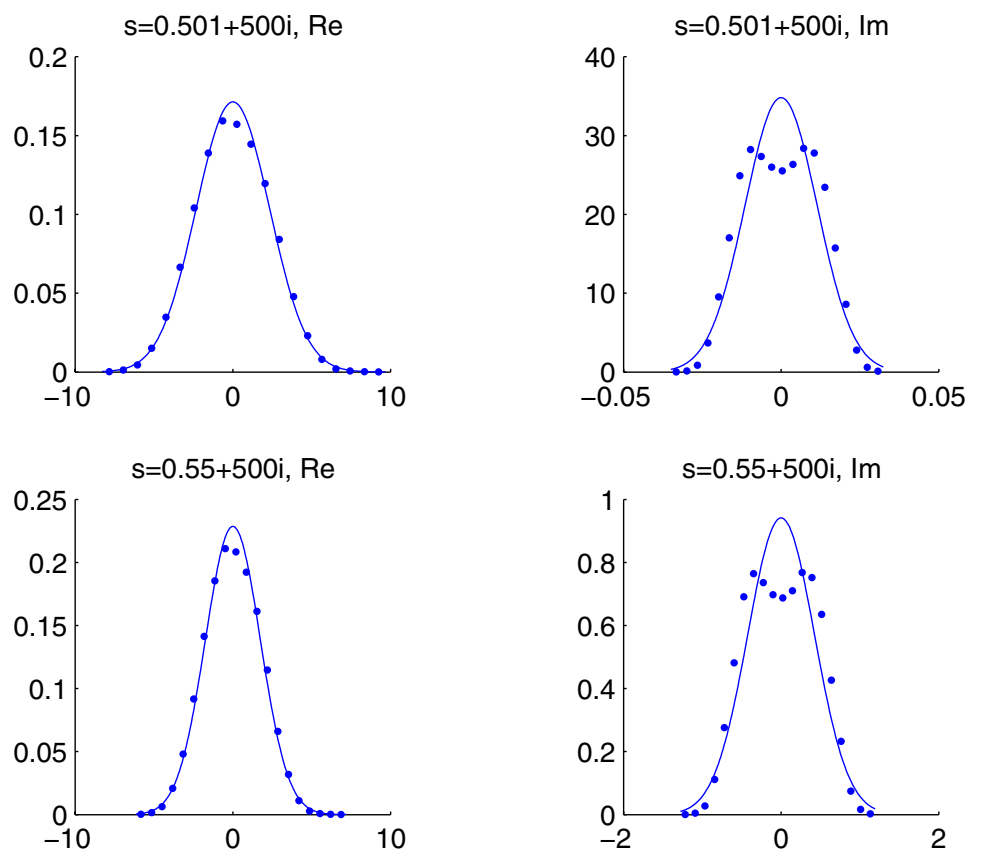

Figure 4. Histograms of the real and imaginary parts of $E^{*}(z ; s)$ for $\widetilde{\Gamma}_{0}(5)$. The solid lines are the Gaussians, and it is clear that $\operatorname{Im} E^{*}(z ; s)$ are far from having Gaussian distribution.
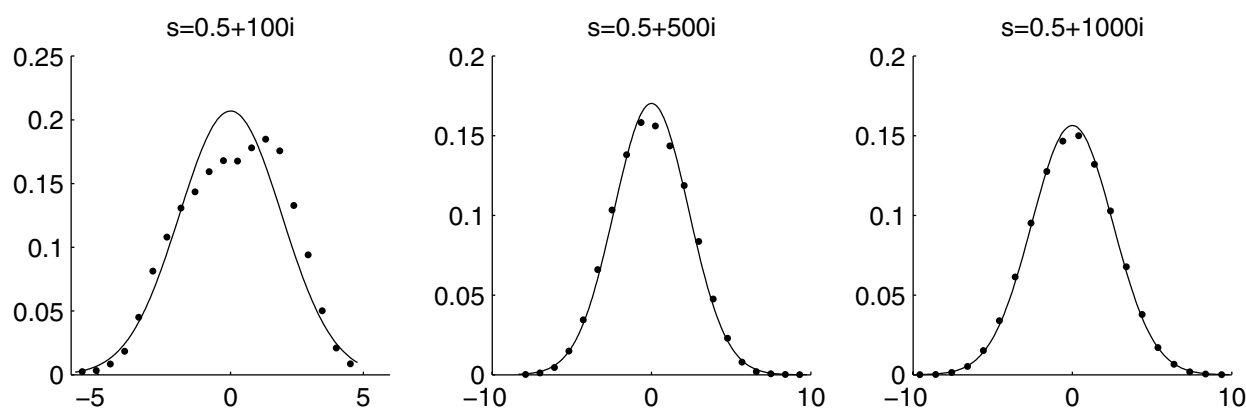

Figure 5. Histograms of $E^{*}(z ; s)$ for $\widetilde{\Gamma}_{0}(5)$. The solid lines are the conjectured Gaussians. 

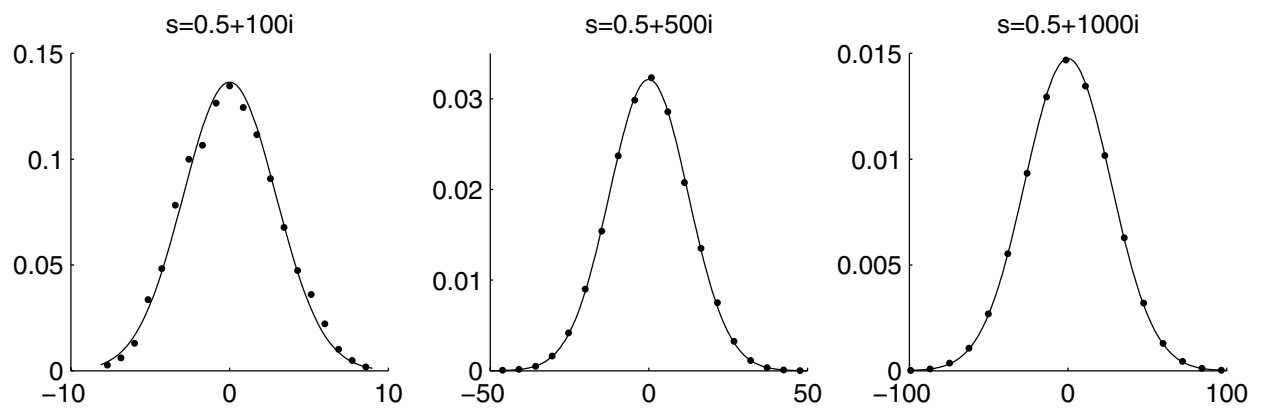

Figure 6 . Histograms of $E^{*}(z ; s)$ for $\Gamma_{1}$. The solid lines are the conjectured Gaussians. The corresponding figure for $\Gamma_{2}$ is of similar quality.

\begin{tabular}{|c|c|ccccc|}
\hline & $\theta$ & $\sigma$ & $\delta$ & $I_{4}$ & $I_{6}$ & $I_{8}$ \\
\hline \hline & 0 & 1.48 & $2 \mathrm{e}-03$ & $6 \mathrm{e}-02$ & $1 \mathrm{e}-01$ & $2 \mathrm{e}-01$ \\
& $\pi / 2$ & 1.35 & $2 \mathrm{e}-03$ & $5 \mathrm{e}-02$ & $1 \mathrm{e}-01$ & $2 \mathrm{e}-01$ \\
& 0.5 & 1.43 & $2 \mathrm{e}-03$ & $5 \mathrm{e}-02$ & $1 \mathrm{e}-01$ & $2 \mathrm{e}-01$ \\
$\Gamma_{1}$ & 0.8 & 1.39 & $1 \mathrm{e}-03$ & $4 \mathrm{e}-02$ & $1 \mathrm{e}-01$ & $2 \mathrm{e}-01$ \\
& 2.0 & 1.40 & $3 \mathrm{e}-03$ & $7 \mathrm{e}-02$ & $1 \mathrm{e}-01$ & $3 \mathrm{e}-01$ \\
& 4.0 & 1.38 & $1 \mathrm{e}-03$ & $4 \mathrm{e}-02$ & $1 \mathrm{e}-01$ & $2 \mathrm{e}-01$ \\
\hline \hline \multirow{5}{*}{$\Gamma_{2}$} & 0 & 1.45 & $2 \mathrm{e}-03$ & $5 \mathrm{e}-02$ & $1 \mathrm{e}-01$ & $2 \mathrm{e}-01$ \\
& $\pi / 2$ & 1.38 & $2 \mathrm{e}-03$ & $5 \mathrm{e}-02$ & $1 \mathrm{e}-01$ & $2 \mathrm{e}-01$ \\
& 0.5 & 1.46 & $2 \mathrm{e}-03$ & $6 \mathrm{e}-02$ & $1 \mathrm{e}-01$ & $2 \mathrm{e}-01$ \\
& 0.8 & 1.44 & $2 \mathrm{e}-03$ & $5 \mathrm{e}-02$ & $1 \mathrm{e}-01$ & $2 \mathrm{e}-01$ \\
& 2.0 & 1.38 & $2 \mathrm{e}-03$ & $6 \mathrm{e}-02$ & $1 \mathrm{e}-01$ & $2 \mathrm{e}-01$ \\
& 4.0 & 1.44 & $2 \mathrm{e}-03$ & $5 \mathrm{e}-02$ & $1 \mathrm{e}-01$ & $2 \mathrm{e}-01$ \\
\hline
\end{tabular}

TABle 10. Statistics for $\cos (\theta) \operatorname{Re} E(z ; s)+\sin (\theta) \operatorname{Im} E(z ; s), s=$ $0.55+1000 i$, indicating a complex Gaussian distribution for $E(z ; s)$ as $\operatorname{Im} s \rightarrow \infty$.

\begin{tabular}{|c|c|ccc|}
\hline & $R$ & $\sigma_{0}$ & $\sigma$ & $\left|\sigma_{0}-\sigma\right| / \sigma_{0}$ \\
\hline \hline \multirow{2}{*}{$\widetilde{\Gamma}_{0}(5)$} & 100 & 1.87 & 1.93 & 0.03 \\
& 500 & 2.25 & 2.34 & 0.04 \\
& 1000 & 2.46 & 2.55 & 0.03 \\
\hline \multirow{3}{*}{$\Gamma_{1}$} & 100 & 3.37 & 2.92 & 0.13 \\
& 500 & 12.99 & 12.41 & 0.05 \\
& 1000 & 25.69 & 27.03 & 0.05 \\
\hline & 100 & 3.12 & 3.21 & 0.03 \\
$\Gamma_{2}$ & 500 & 10.27 & 9.75 & 0.05 \\
& 1000 & 11.99 & 12.86 & 0.07 \\
\hline
\end{tabular}

TABLE 11. Comparison between standard deviation $\sigma_{0}$ as predicted by HR92 and standard deviation $\sigma$ computed from our data. 
Finally, we give some data in connection with a conjectural asymptotic formula for the standard deviation $\sigma$ over a given rectangle $F$, based on [HR92. Let $y_{a v}$ be the average $y$-value over $F$ and set

$$
\begin{gathered}
N=\frac{R}{2 \pi y_{a v}}, \quad \Omega=\frac{2}{N} \sum_{1 \leq|m| \leq N}\left|\varphi_{m}(s)\right|^{2}, \\
\sigma_{0}^{2}=\sigma_{1}^{2}+\frac{\pi \Omega}{8}, \quad \sigma_{1}^{2}=\frac{1}{\mu(F)} \int_{F}\left|y^{\frac{1}{2}+i R}+\varphi\left(\frac{1}{2}+i R\right) y^{\frac{1}{2}-i R}\right|^{2} d \mu .
\end{gathered}
$$

In line with the heuristic arguments in [HR92, $\S \S 6,7]$ it is natural to expect that we should have $\sigma \approx \sigma_{0}$ as $R \rightarrow \infty$. One also expects that $\sigma_{1}$ should be asymptotically negligible in the limit, thus $\sigma \approx \sqrt{\pi \Omega / 8}$ as $R \rightarrow \infty$. We test this conjecture in Table 11 where we see that we already have a reasonable agreement for our fairly modest $R$-values.

Recall that [HR92, §7] dealt with the arithmetic group $\Gamma=P S L(2, \mathbb{Z})$, and it was seen that in this case the term $c_{1} N \log N$ is dominant in (8) when $N=R /\left(2 \pi y_{a v}\right)$ and $R \rightarrow \infty$, leading to

$$
\Omega \sim \frac{16 \log R}{\pi \mu(\Gamma \backslash \mathcal{H})}=\frac{48 \log R}{\pi^{2}}
$$

and the expected asymptotic formula $\sigma \sim \sqrt{6 \log R / \pi}$. However, we remark that the asymptotic size of $\Omega$ may well look quite different on a generic group: First of all, by the Phillips-Sarnak philosophy, we expect the term $\varphi^{\prime}\left(\frac{1}{2}+i R\right) / \varphi\left(\frac{1}{2}+i R\right)$ in $c_{2}$ to frequently be of size at least [const] $R$, thus making $c_{2} N$ dominate heavily over $c_{1} N \log N$ in (8); but furthermore it is not clear if the implied constant in the error term in (8) can be bounded with respect to $R$ in a way so that the estimate is at all relevant when $N=R /\left(2 \pi y_{a v}\right)$.

These experiments have only begun to uncover the true nature of the value statistics of $E(z ; s)$. A number of intriguing questions remain to be investigated, both numerically and theoretically. On non-arithmetic groups we see a clear tendency of a Gaussian limit distribution for our Re $s$-values, and it is not unreasonable to believe that we have a Gaussian limit as $\operatorname{Im} s \rightarrow \infty$, for $\operatorname{Re} s \geq 1 / 2$ near $1 / 2$. It is not clear what the exact limitations on $\operatorname{Re} s$ should be for this to hold. Would it perhaps be true for all $1 / 2 \leq \operatorname{Re} s<1$ ?

Regarding the arithmetic group, one can speculate if $E(z ; s)$ has a complex Gaussian distribution there as well as $\operatorname{Im} s \rightarrow \infty$, for $\operatorname{Re} s>1 / 2$ with $\operatorname{Re} s \rightarrow 1 / 2$, say. This might very well be the case, although the evidence is not accessible for our values of $\operatorname{Im} s$. It would certainly be interesting to study theoretically in what Re $s$-regime the phenomenon displayed in Figure 4 persists as $\operatorname{Im} s \rightarrow \infty$. This would in particular involve studying the asymptotics of $K_{s-1 / 2}(X)$. 
3.4. Pictures of $E(z ; s)$. We end this paper by showing pictures of $E(x+i y ; s)$ in $-0.75<x<0.75,0.15<y<0.65$. We have computed values at $500 \times 500$ points and given them colors ranging through blue, green, yellow and red as the values pass from their minimum to their maximum.

Figure 7 is for the arithmetic group $\widetilde{\Gamma}_{0}(5)$. The upper two plots are of the real valued $e^{-i \omega / 2} E(z ; s)$ for $\operatorname{Re} s=1 / 2$ and $\operatorname{Im} s=16, \operatorname{Im} s=100$. The next two plots in the left hand column are of $\operatorname{Re} E(z ; s)$ and $\operatorname{Im} E(z ; s)$ with $s=0.55+16 i$. The last two plots in the right hand column are the real and imaginary parts of $e^{-i \omega_{1}} E(z ; s)$ for $s=0.501+100 i$, and here we note the "linear pattern" for the imaginary part redisplaying the phenomenon in Figure 4 discussed in section 3.3 .

In Figure 8 we have $\Gamma_{1}$ in the left hand column and $\Gamma_{2}$ in the right hand column and $s$-values $0.5+16 i$ and $0.55+16 i$. The Eisenstein series on non-arithmetic

groups show a more chaotic behavior than on $\widetilde{\Gamma}_{0}(5)$, and this nicely illustrates our findings in section 3.3 . 

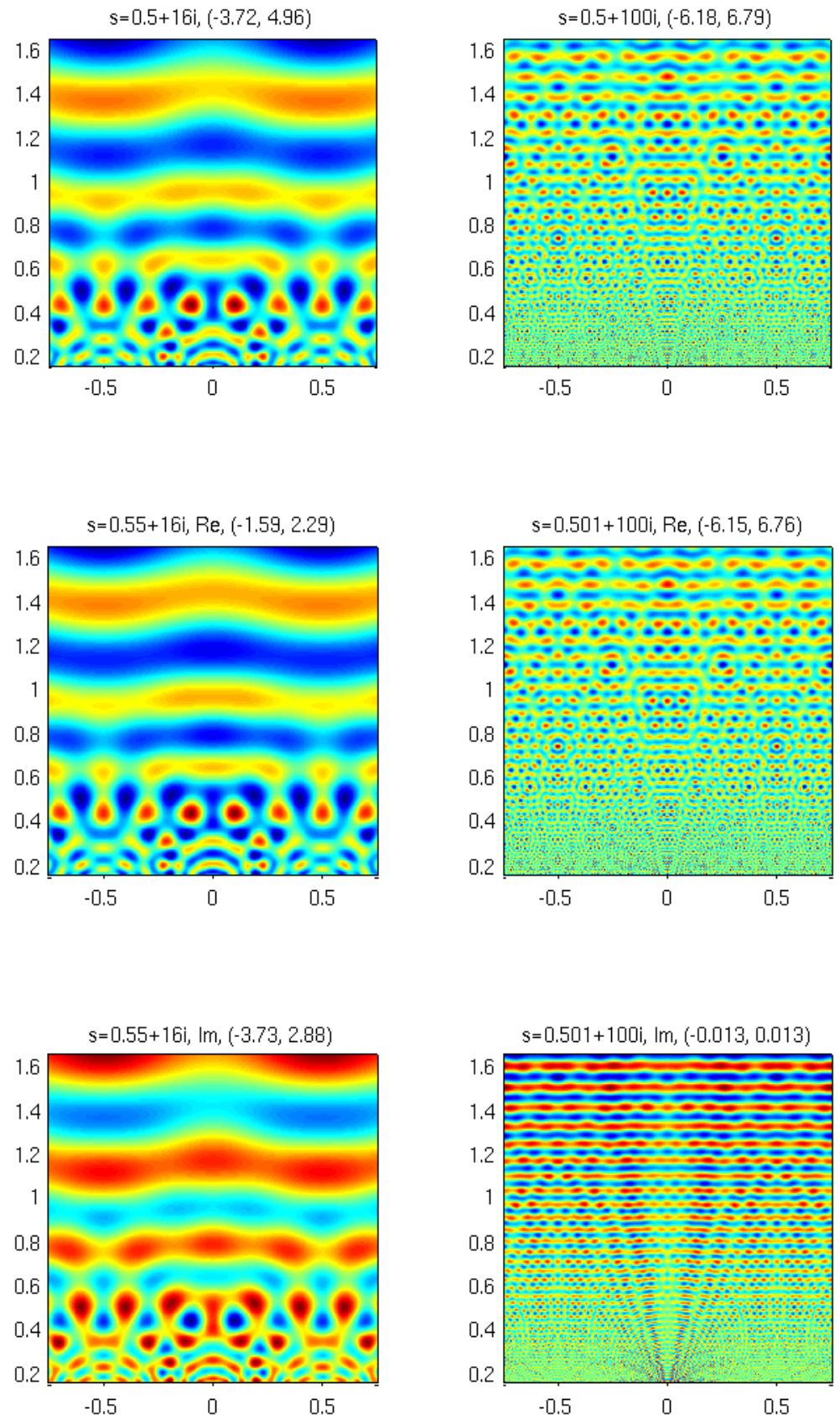

FiguRE 7. Pictures of the Eisenstein series on the arithmetic group $\widetilde{\Gamma}_{0}(5)$. Minimum and maximum values are given in parentheses. 

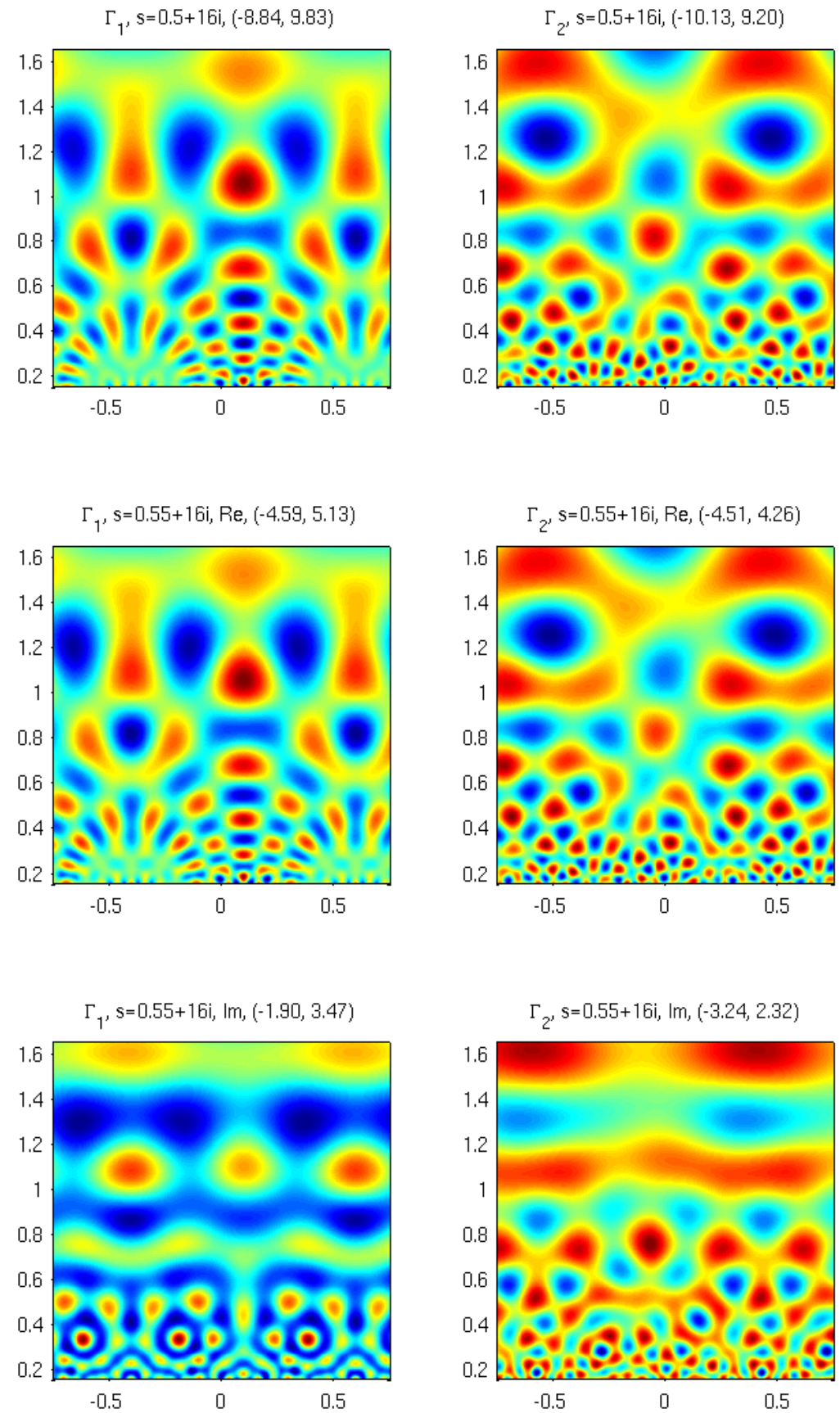

Figure 8. Pictures of the Eisenstein series, $E^{*}(z ; s)$. Minimum and maximum values are given in parentheses. 


\section{ACKNOWLEDGMENTS}

The author is most grateful to Andreas Strömbergsson, Dennis Hejhal, Fredrik Strömberg and Stefan Lemurell for helpful discussions on this work.

\section{REFERENCES}

[Ave03] H. Avelin, On the deformation of cusp forms, U.U.D.M. Report 2003:8, Uppsala, 85 pp. http://www.math.uu.se/research/pub/Avelin2.pdf, 2003.

[Ave04] - Some computations used to prove that $\Gamma_{a, r}$ is a Fuchsian group, 2004, Maple-file http://www . math.uu.se/research/archive/avelin.

[Ave07] —, Deformation of $\Gamma_{0}(5)$-cusp forms, Math. Comp. 76 (2007), 361-384. MR 2261026

[Ber72] L. Bers, Uniformization, moduli, and Kleinian groups, Bull. London Math. Soc. 4 (1972), 257-300. MR0348097 (50:595)

[Ber77] M. V. Berry, Regular and irregular semiclassical wavefunctions, J. Phys. A: Math. Gen. 10 (1977), no. 12, 2083-2091. MR0489542 (58:8961)

[BR99] J. Bernstein and A. Reznikov, Analytic continuation of representations and estimates of automorphic forms, Ann. of Math. 150 (1999), no. 1, 329-352. MR.1715328 (2001h:11053)

[Col85] Y. Colin de Verdiere, Ergodicité et fonctions propres du laplacien, Comm. Math. Phys. 102 (1985), 497-502. MR818831 (87d:58145)

[FL05] D. W. Farmer and S. Lemurell, Deformations of Maass forms, Math. Comp. 74 (2005), no. 252, 1967-1982. MR2164106 (2007a:11060)

[Gut95] A. Gut, An intermediate course in probability, Springer-Verlag, New York, 1995. MR.1353911 (97c:60001)

[Hej83] D. A. Hejhal, The Selberg Trace Formula for PSL $(2, \mathbb{R})$, vol. 2, Lecture Notes in Math. 1001, Springer-Verlag, Berlin, 1983. MR711197 (86e:11040)

[Hej99] _ - On eigenfunctions of the Laplacian for Hecke triangle groups, Emerging Applications of Number Theory (D. Hejhal, J. Friedman, M. Gutzwiller, and A. Odlyzko, eds.), Springer-Verlag, 1999, pp. 291-315. MR1691537 (2000f:11063)

[Hel66] H. Helling, Bestimmung der Kommensurabilitätsklasse der Hilbertschen Modulgruppe, Math. Z. 92 (1966), 269-280. MR0228437 (37:4017)

[Hop36] E. Hopf, Fuchsian groups and ergodic theory, Trans. Amer. Math. Soc. 39 (1936), no. 2, 299-314. MR.1501848

[HR92] D. A. Hejhal and B. N. Rackner, On the Topography of Maass Waveforms for PSL(2, Z), Exp. Math. 1 (1992), no. 4, 275-305. MR1257286 (95f:11037)

[HS01] D. A. Hejhal and A. Strömbergsson, On quantum chaos and Maass waveforms of CMtype, Found. Phys. 31 (2001), no. 3, 519-533. MR1839791 (2003k:81066)

[Lin06] E. Lindenstrauss, Invariant measures and arithmetic quantum unique ergodicity, Ann. of Math. 163 (2006), no. 1, 165-219. MR2195133 (2007b:11072)

[LS95] W. Luo and P. Sarnak, Quantum ergodicity of eigenfunctions on $P S L_{2}(\mathbf{Z}) \backslash \mathbf{H}^{2}$, Publ. Math. IHES 81 (1995), 207-237. MR1361757 (97f:11037)

[LS03] _ Mass equidistribution for Hecke eigenforms, Comm. Pure Appl. Math. LVI (2003), 0874-0891. MR 1990480 (2004e:11038)

[LS04] , Quantum variance for Hecke eigenforms, Ann. Sci. École Norm. Sup. 37 (2004), 769-799. MR2103474 (2005k:11101)

[MS83] C. J. Moreno and F. Shahidi, The fourth moment of Ramanujan $\tau$-function, Math. Ann. 266 (1983), 233-239. MR724740 (85i:11039)

[PS85] R. S. Phillips and P. Sarnak, On cusp forms for cofinite subgroups of $P S L(2, \mathbb{R})$, Invent. Math. 80 (1985), 339-364. MR788414 (86m:11037)

[Sar01] P. Sarnak, Estimates for Rankin-Selberg L-functions and quantum unique ergodicity, J. Funct. Anal. 184 (2001), 419-453. MR1851004 (2003c:11050)

[Sel89] A. Selberg, Göttingen Lectures on Harmonic Analysis, in Collected Papers, vol. 1, Springer-Verlag, 1989, pp. 626-674.

[Shn74] A. I. Shnirelman, Ergodic properties of eigenfunctions, Uspekhi Mat. Nauk. 29 (1974), no. 6, 181-182. MR0402834 (53:6648) 
[Str05a] A. Strömbergsson, On the Fourier coefficients of Eisenstein series for the hyperbolic plane, in preparation, 2005.

[Str05b] - Remarks on moments of divisor functions, personal communication, 2005.

[The05] H. Then, Maass cusp forms for large eigenvalues, Math. Comp. 74 (2005), 363-381. MR:2085897(2005h:11106)

[Zel87] S. Zelditch, Uniform distribution of eigenfunctions on compact hyperbolic surfaces, Duke Math. J. 55 (1987), no. 4, 919-941. MR916129 (89d:58129)

[Zel91] _ Mean Lindelöf hypothesis and equidistribution of cusp forms and Eisenstein series, J. Funct. Anal. 97 (1991), no. 1, 1-49. MR1105653 (92h:11046)

Department of Mathematics, Uppsala University, S-751 06 Uppsala, Sweden

E-mail address: helen.avelin@math.uu.se 